\title{
Bethe Ansatz solutions for Temperley-Lieb Quantum Spin Chains
}

\author{
R. C. T. Ghiotto and A. L. Malvezzi \\ Universidade Estadual Paulista - UNESP Faculdade de Ciências Departamento de \\ Física Caixa Postal 473, CEP 17033-360 Bauru, SP, Brazil
}

\begin{abstract}
We solve the spectrum of quantum spin chains based on representations of the TemperleyLieb algebra associated with the quantum groups $\mathcal{U}_{q}\left(X_{n}\right)$ for $X_{n}=A_{1}, B_{n}, C_{n}$ and $D_{n}$. The tool is a modified version of the coordinate Bethe Ansatz through a suitable choice of the Bethe states which give to all models the same status relative to their diagonalization. All these models have equivalent spectra up to degeneracies and the spectra of the lower dimensional representations are contained in the higher-dimensional ones. Periodic boundary conditions, free boundary conditions and closed non-local boundary conditions are considered. Periodic boundary conditions, unlike free boundary conditions, break quantum group invariance. For closed non-local cases the models are quantum group invariant as well as periodic in a certain sense.
\end{abstract}




\section{Introduction}

Temperley-Lieb (TL) algebra [1] has been widely used in the construction of solutions of the Yang-Baxter equation [2, 3], which is a sufficient condition for integrability of lattice models through the quantum inverse scattering method. On the other hand, quantum group [4 in some cases is a symmetry of the integrable model [5]. Thus, quantum group together with Temperley-Lieb algebra provide a powerful algebraic framework to build and study various kinds of lattice models in two-dimensional statistical mechanics. One particular way of building models which are quantum group invariant uses the TL algebra. It is a algebra generated by the Hamiltonian density $U_{k}, k=1,2, \ldots, N-1$ subject to the following constraints

$$
\begin{array}{ll}
U_{k}^{2}=\left(Q+Q^{-1}\right) U_{k} & , \quad U_{k} U_{k \pm 1} U_{k}=U_{k} \\
U_{k} U_{j}=U_{j} U_{k} & |k-j|>1 .
\end{array}
$$

with $Q \in C$ a given number. This algebra appears in a large class of quantum lattice models and leads, at level of free and ground state energies, to some equivalence among the models [2].

Taking into account usual boundary conditions, the TL Hamiltonians take the form

$$
\mathcal{H}=\sum_{k=1}^{N-1} U_{k k+1}+\mathbf{b t}
$$

where $U_{k k+1} \equiv U_{k}$ operates in a direct product of complex spaces at positions $k$ and $k+1$. In general, they are not invariant with respect to quantum groups since the boundary terms, bt, break translational invariance, reflecting the non-cocommutativity of the co-product. Indeed, we know from [6, 4, 7] that very special boundary terms must be considered when we seek these quantum group invariant spin chains. In particular, one possibility is to consider free boundary conditions, i.e., bt $=0$. For The XXZ-Hamiltonian with free boundary conditions one has to apply the Bethe ansatz [8] techniques introduced first by Alcaraz et al [6] and after by Sklyanin [9] using Cherednik's reflection matrices [10, 11]. By this method the XXZ-Heisenberg model [12], the $\operatorname{spl}_{q}(2,1)$ invariant supersymmetric t-J model [13, 14] , the $U_{q}[\operatorname{sl}(n)]$ invariant generalization of the XXZ-chain [15] and the $S U_{q}(n \mid m)$ spin chains [7, 16] have been solved for free boundary conditions.

Recently, by means of a generalized algebraic nested Bethe ansatz, Karowski and Zapletal [17] presented a class of quantum group invariant $n$-state vertex models with periodic boundary conditions. Also an extension of this method to the case of graded vertex models was analyzed in [18], where a $s p l_{q}(2 \mid 1)$ invariant susy $t-J$ model with closed boundary conditions was presented.

In fact, these kind of models were first discussed by Martin [19] from the representations of the Hecke algebra. In this case, the boundary term is a non-local operator, bt $=\mathcal{U}_{0}$ (see Section 4 ). The study of closed quantum group invariant closed spin chains in the framework of the coordinate Bethe ansatz was presented by Grosse et al [20] for the fundamental representation of $S U_{q}(2)$ and generalized by [21]. In this context it would be interesting to discuss other quantum group invariant closed spin chains.

In the present paper we find the spectrum of Hamiltonians based on representations of the TL algebra associated with quantum groups. The Bethe ansatz equations for different types of boundary conditions (periodic, closed and free) are obtained through a modified version of the coordinate Bethe ansatz. Therefore we generalize the results of ref. [21]. Our method was recently applied to solve graded T-L Hamiltonian [22] and anisotropic correlated electron model associated with this algebra [23].

The paper is organized as follows. In Section 2, we describe the representations of the TL algebra,

constructed as projectors on total spin zero of two neighboring spins. In Section 3, we introduce the 
modified coordinate Bethe Ansatz for the TL Hamiltonians with periodic boundary conditions. In Section 4 their Bethe Ansatz solution is presented with non-local boundary conditions. In section 5, contains the solution for free boundary conditions. Finally the conclusions are reserved for section 6 .

\section{Representations of the TL algebra}

Representations of the TL algebra, commuting with quantum groups, can be constructed in the following way [24]. Suppose $\mathcal{U}_{q}\left(X_{n}\right)$ is the universal enveloping algebra of a finite dimensional Lie algebra $X_{n}$, equipped with the coproduct $\Delta: \mathcal{U}_{q} \rightarrow \mathcal{U}_{q} \otimes \mathcal{U}_{q}$ [25]. If now $\pi: \mathcal{U}_{q} \rightarrow$ End $V_{\Lambda}$ is a finite dimensional irreducible representation with highest weight $\Lambda$ and we assume that the decomposition $V_{\Lambda} \otimes V_{\Lambda}$ is multiplicity free and includes one trivial representation on $V_{0}$, then the projector $\mathcal{P}_{0}$ from $V_{\Lambda} \otimes V_{\Lambda}$ onto $V_{0}$ is a representation of the $\mathrm{TL}$ algebra. The deformation parameter $q$, which plays the role of a coupling constant in the Hamiltonian, is related to $Q$ as:

$$
Q+Q^{-1}=\operatorname{Tr}_{V_{\Lambda}}\left(q^{-2 \rho}\right)
$$

where $\rho$ is half the sum of the positive roots.

We will consider the following specific cases, $\left(V_{\Lambda}, \mathcal{U}_{q}\left(X_{n}\right)\right)=\left(V_{2 s \Lambda_{1}}, \mathcal{U}_{q}\left(A_{1}\right)\right)$ for spin $s,\left(V_{\Lambda_{1}}, \mathcal{U}_{q}\left(B_{n}\right),\left(V_{\Lambda_{1}}, \mathcal{U}_{q}(C\right.\right.$ and $\left(V_{\Lambda_{1}}, \mathcal{U}_{q}\left(D_{n}\right)\right.$. Namely we treat the $q$-deformations of the spin-s representation of $s l(2)$ and the vector representations of $s o(2 n+1), s p(2 n)$ and $s o(2 n)$. $V_{\Lambda}$ denotes the $\mathcal{U}_{q}\left(X_{n}\right)$ module with highest weight $\Lambda$ and $\Lambda_{1}$ is a highest weight of $X_{n}$.

Since we are not going to use any group-theoretical machinery, we will just lift the relevant formulas off Batchelor and Kuniba [3] in order to display explicitly the Hamiltonians to be diagonalized.

We introduce orthonormal vectors $e_{\mu},<e_{\mu}, e_{\nu}>=\delta_{\mu \nu}$, to express $\rho$ and the set $\mathcal{A}$ of weights appearing in the representation $\pi$ of $\mathcal{U}_{q}\left(X_{n}\right)$ as follows:

$A_{1}$ : Spin-s representation of the $s l(2)$ algebra

$$
\begin{gathered}
\mathcal{A}=\left\{s e_{12},(s-1) e_{12}, \ldots,-s e_{12}\right\}, \quad \rho=\frac{1}{2} e_{12}, \quad\left(e_{12}=e_{1}-e_{2}\right), \\
J=\{s, s-1, \ldots,-s\}, \quad \epsilon(\mu)=(-1)^{\tilde{\mu}}, \quad Q+Q^{-1}=[2 s+1] .
\end{gathered}
$$

$B_{n}:$ Vector representation of the $s o(2 n+1)$ algebra $(n \geq 2)$

$$
\begin{array}{r}
\mathcal{A}=\left\{0, \pm e_{1}, \ldots, \pm e_{n}\right\}, \rho=\sum_{\alpha=1}^{n}\left(n-\left(\alpha-\frac{1}{2}\right)\right) e_{\alpha}, J=\{0, \pm 1, \ldots, \pm n\} \\
\epsilon(\mu)=(-1)^{\tilde{\mu}}, \quad Q+Q^{-1}=\frac{[2 n-1][n+1 / 2]}{[n-1 / 2]} .
\end{array}
$$

$C_{n}:$ Vector representation of the $\operatorname{sp}(2 n)$ algebra $(n \geq 1)$

$$
\begin{array}{r}
\mathcal{A}=\left\{ \pm e_{1}, \ldots, \pm e_{n}\right\}, \quad \rho=\sum_{\alpha=1}^{n}(n-\alpha+1) e_{\alpha}, \quad J=\{ \pm 1, \ldots, \pm n\} \\
\epsilon(\mu)=\operatorname{sign}(\mu), \quad Q+Q^{-1}=\frac{[n][2 n+2]}{[n+1]} .
\end{array}
$$


$D_{n}$ : Vector representations of the $s o(2 n)$ algebra $(n \geq 3)$

$$
\begin{aligned}
\mathcal{A}=\left\{ \pm e_{1}, \ldots, \pm e_{n}\right\}, \quad \rho=\sum_{\alpha=1}^{n-1}(n-\alpha) e_{\alpha}, \quad J=\{ \pm 1, \ldots, \pm n\}, \\
\epsilon(\mu)=1, \quad Q+Q^{-1}=\frac{[2 n-2][n]}{[n-1]} .
\end{aligned}
$$

For $\mu \in J$ the symbol $\tilde{\mu}$ is defined as $\tilde{\mu}=\mu+1 / 2$ for $A_{1}$ with $s$ semi-integer and $\tilde{\mu}=\mu$ for $A_{1}$ with $s$ integer. For $B_{n}, C_{n}$ and $D_{n}, \tilde{\mu}=0$ with the exception of $\tilde{0}=1$ for $B_{n}$. The $q$-number notation is $[x]=\left(q^{x}-q^{-x}\right) /\left(q-q^{-1}\right)$.

For $X_{n}=B_{n}, C_{n}$ and $D_{n}$, we extend the suffix of $e_{\mu}$ to $-n \leq \mu \leq n$ by setting $e_{-\mu}=-e_{\mu}$ (hence $\left.e_{0}=0\right)$. Using the index set $J$ above, we can write $\mathcal{A}=\left\{\mu\left(e_{1}-e_{2}\right)\right\}$ for $A_{1}$ and $\left.\mathcal{A}=\left\{e_{\mu} \mid \mu \in J\right)\right\}$ for $B_{n}, C_{n}$ and $D_{n}$.

Denoting by $E_{\mu \nu} \in$ End $V_{\Lambda}$ the matrix unit, having all elements zero, except at row $\mu$ and column $\nu$, the projector can be written as

$$
\mathcal{P}_{0}=\frac{1}{Q+Q^{-1}} \sum_{\mu, \nu \in J} \epsilon(\mu) \epsilon(\nu) q^{-<e_{\mu}+e_{\nu}, \rho>} E_{\mu \nu} \otimes E_{-\mu-\nu} .
$$

In the following we will refer to all models generically as higher spin models for simplicity, even when not talking about $A_{1}$. The dimensions of $B_{n}$ are related with the dimensions of $A_{1}$ for $s$ integer and the dimensions of $C_{n}$ and $D_{n}$ are related with the dimensions of $A_{1}$ for $s$ semi-integer.

If we consider then a one-dimensional chain of length $N$ with a "spin" at each site, the "spin variables " range over the set of weight vectors $\mathcal{A}=\left\{v_{\mu} \mid \mu \in J\right\}$ and our Hilbert space is an $N$-fold tensor product $V_{\Lambda} \otimes \ldots \otimes V_{\Lambda}$. For $A_{1}$, these are the $q$-analogs of the usual spin states. The periodic Hamiltonians associated with the TL representations are given by the following sum over $N$ sites

$$
\mathcal{H}=\sum_{k=1}^{N} U_{k} .
$$

Here $\mathbf{b t}=U_{N, N+1}=U_{N 1}$ and the Hamiltonian densities acting on two neighboring sites are then given by:

$$
\begin{aligned}
\langle\nu, \lambda|U| \mu, \kappa\rangle & =\epsilon(\mu) \epsilon(\nu) q^{-\left\langle e_{\mu}+e_{\nu}, \rho\right\rangle} \delta_{\mu+\kappa, 0} \delta_{\nu+\lambda, 0} \\
\mu, \nu, \kappa, \lambda & \in J
\end{aligned}
$$

Using representations of the TL algebra, one can also build solvable vertex models whose Hamiltonian limit leads to the previously mentioned quantum spin chains. To do so, we introduce an operator $R(u) \in$ End $\left(V_{\Lambda} \otimes V_{\Lambda}\right)$ by

$$
\begin{gathered}
R_{k}(u)=I \otimes \cdots \otimes I \otimes \underbrace{R(u)}_{k, k+1} \otimes I \otimes \cdots \otimes I \\
R(u)=\sum_{\mu, \nu, \kappa, \lambda \in J} R_{\mu \kappa}^{\nu \lambda}(u, \eta) E_{\mu \nu}^{(k)} \otimes E_{\kappa \lambda}^{(k+1)} \\
R_{\mu \kappa}^{\nu \lambda}(u, \eta)=\frac{\sinh (\eta-u)}{\sinh \eta} \delta_{\mu \nu} \delta_{\kappa \lambda}+\frac{\sinh u}{\sinh \eta} \epsilon(\mu) \epsilon(\nu) q^{-<e_{\mu}+e_{\nu}, \rho>} \delta_{\mu+\kappa, 0} \delta_{\nu+\lambda, 0}
\end{gathered}
$$


where $u$ is the spectral parameter and the anisotropy parameter $\eta$ is chosen so that

$$
2 \cosh \eta=Q+Q^{-1}
$$

The $R$ matrix commutes with the quantum group action and the Yang-Baxter equation

$$
R_{k}(u) R_{k+1}(u+v) R_{k}(v)=R_{k+1}(v) R_{k}(u+v) R_{k+1}(u)
$$

is valid owing to the $T L$ relations (1.1).

Due to the TL algebra these models are equivalent to the 6 -vertex and the " $p$-state " self-dual Potts models $\left(\sqrt{p}=\lim _{q \rightarrow 1}\left(Q+Q^{-1}\right)\right)$ through the argument in [2]. In fact, the cases $\mathcal{U}_{q}\left(A_{1}\right)$ with $s=1 / 2$ and $\mathcal{U}_{q}\left(C_{1}\right)$ yield the 6 -vertex model itself. When $q=1$, the vertex models here reduce to those discussed in [26, 27, 28], where the number of states is equal to $\sqrt{p}$.

The case $A_{1}$ have been studied by several authors. When $q=1$ and $s=1 / 2$ the operator $U_{k}$ is essentially the Heisenberg interaction term $\sigma_{k}^{x} \sigma_{k+1}^{x}+\sigma_{k}^{y} \sigma_{k+1}^{y}+\sigma_{k}^{z} \sigma_{k+1}^{z}$. Besides the explicit matrix elements, available from (2.8), the local Hamiltonian $U_{k}$ is in principle also expressible in terms of the usual representation matrices for $S U(2)$ generators. The resulting $q$-deformed Hamiltonian has been written down for $s=1$ in [5]. However for $s>1$, writing the Hamiltonian in terms of the usual $S U(2)$ operators becomes very cumbersome. These Hamiltonians have alternative expressions in terms of Casimir operators [3].

The limit $q \rightarrow 1$ has discussed for general $s$ [29, 30]. As for related spin-1 biquadratic model [29, 31], they are massive for $s \geq 1$ and of relevance to the dimerization transition on $S U(n)$ antiferromagnetic chains [32]. The case $s=1 / 2$ has also been investigated in some detail in [4].

Having now built common ground for all models, whose salient feature is they being spin zero projectors, we may now follow the steps of reference [33] to find their spectra.

\section{Bethe Ansatz: Periodic boundary conditions}

All the above Hamiltonians are $U(1)$ invariant and we can classify their spectra according to sectors. For $A_{1}$ the commuting operator is the total spin $S^{z}=\sum_{k=1}^{N} S_{k}^{z}$ where $S_{k}^{z}=\operatorname{diag}(s, s-1, \ldots,-s+1,-s)_{k}$ and we set the conserved quantum number $r=s N-S^{z}$. We extend these quantum numbers for the other algebras as $r=N \omega-\mathbf{S}^{z}$ where $\mathbf{S}^{z}=\sum_{k} \mathbf{S}_{k}^{z}$ with $\mathbf{S}_{k}^{z}=\operatorname{diag}(\omega, \omega-1, \ldots,-\omega+1,-\omega)_{k}$ and $\omega=\max \{J\}$. Eigenvalues of the operator $r$ can be used to collect the eigenstates of $\mathcal{H}$ in sectors, $\Psi_{r}$. Therefore, there exists a reference state $\Psi_{0}$, satisfying $\mathcal{H} \Psi_{0}=E_{0} \Psi_{0}$, with $E_{0}=0$. We take $\Psi_{0}$ to be $\Psi_{0}=|\omega \omega \omega \cdots \omega\rangle$. It is the only eigenstate in the sector $r=0$ and all other energies will be measured relative to this state.

In every sector $r$ there are eigenstates degenerate with $\Psi_{0}$. They contain a set of impurities. We call impurity any state obtained by lowering some of the $|\omega, k\rangle$ 's, such that the sum of any two neighboring spins is non-zero. Since $\mathcal{H}$ is a projector on spin zero, all these states are annihilated by $\mathcal{H}$. In particular, they do not move under the action of $\mathcal{H}$, which is the reason for their name.

Nothing interesting happens in sectors with $r<2 \omega$. Sector $r=2 \omega$, we encounter the situation where the states $|\alpha, k\rangle$ and $|-\alpha, k \pm 1\rangle, \alpha \in J$, occur in neighboring pairs. They move under the action of $\mathcal{H}$, i.e., the sector $r=2 \omega$ contains one free pseudoparticle. In general, for a sector $r$ we may have $p$ pseudoparticles and $N_{\omega-1}, N_{\omega-2}, \ldots, N_{-\omega+1}$ impurities of the type $\omega-1, \omega-2, \ldots,-\omega+1$, respectively, such that

$$
r=2 \omega p+\sum_{\alpha=1}^{2 \omega-1} \alpha N_{\omega-\alpha}
$$


The main result of this section is to show that $\mathcal{H}$ can be diagonalized in a convenient basis, constructed from products of single pseudoparticle wavefunctions. The energy eigenvalues will be parametrized as a sum of single pseudoparticle contributions.

The first nontrivial sector is $r=2 \omega$ and the correspondent eigenspace is spanned by the states $|k(-\alpha, \alpha)>=| \omega \omega \cdots \omega-\alpha \alpha \omega \cdots \omega>$, where $k=1,2, \ldots, N-1$ and $\alpha \in J$. We seek eigenstates of $\mathcal{H}$ which are linear combinations of these vectors. It is very convenient to consider the linear combination

$$
|\Omega(k)\rangle=\sum_{\alpha=-\omega}^{\omega} \epsilon(\omega) \epsilon(\alpha) q^{-<e_{\omega}+e_{\alpha}, \rho>}|k(-\alpha, \alpha)\rangle,
$$

which is an eigenstate of $U_{k}$ :

$$
U_{k}|\Omega(k)\rangle=\left(Q+Q^{-1}\right)|\Omega(k)\rangle .
$$

Moreover, the action of $U_{k \pm 1}$ on $|\Omega(k)\rangle$ is very simple

$$
\begin{array}{ll}
U_{k-1}|\Omega(k)\rangle=\epsilon|\Omega(k-1)\rangle & U_{k+1}|\Omega(k)\rangle=\epsilon|\Omega(k+1)\rangle \\
U_{k}|\Omega(m)\rangle=0 & k \neq\{m \pm 1, m\}
\end{array}
$$

where $\epsilon=1$ for $B_{n}, D_{n}$ and $A_{1}$ ( $s$ integer) and $\epsilon=-1$ for $C_{n}$ and $A_{1}$ ( $s$ semi-integer).

It should be emphasized that although the linear combination (3.2) is different for each model, the action of $U_{k}$ is always given by (3.3) and (3.4). Therefore, all Hamiltonians can be treated in a similar way and it affords a considerable simplification in their diagonalizations when we compare with the calculus used in the usual spin basis [33].

\subsection{One-pseudoparticle}

We will now start to diagonalize $\mathcal{H}$ in every sector. Let us consider one free pseudoparticle as a highest weight state which lies in the sector $r=2 \omega$

$$
\Psi_{2 \omega}=\sum_{k} A(k)|\Omega(k)\rangle \text {. }
$$

Using the eigenvalue equation $\mathcal{H} \Psi_{2 \omega}=E_{2 \omega} \Psi_{2 \omega}$, one can derive a complete set of equations for the wavefunctions $A(k)$.

When the bulk of $\mathcal{H}$ acts on $|\Omega(k)\rangle$ it sees the reference configuration, except in the vicinity of $k$ where we use (3.3) and (3.4) to get

$$
\begin{array}{r}
\mathcal{H}|\Omega(k)\rangle=\left(Q+Q^{-1}\right)|\Omega(k)\rangle+\epsilon|\Omega(k-1)\rangle+\epsilon|\Omega(k+1)\rangle \\
2 \leq k \leq N-2
\end{array}
$$

Substituting (3.6) in the eigenvalue equation, we get

$$
\begin{array}{r}
\left(E_{2 \omega}-Q-Q^{-1}\right) A(k)=\epsilon A(k-1)+\epsilon A(k+1) \\
2 \leq k \leq N-2
\end{array}
$$

Here we will treat periodic boundary conditions. They demand $U_{N, N+1}=U_{N, 1}$, implying $A(k+N)=$ $A(k)$. This permits us to complete the set of equations (3.7) for $A(k)$ by including the equations for 
$k=1$ and $k=N-1$. Now we parametrize $A(k)$ by plane wave $A(k)=A \xi^{k}$ to get the energy of one free pseudoparticle as:

$$
\begin{array}{r}
E_{2 \omega}=Q+Q^{-1}+\epsilon\left(\xi+\xi^{-1}\right) \\
\xi^{N}=1
\end{array}
$$

Here $\xi=\mathrm{e}^{i \theta}, \theta$ being the momenta determined from the periodic boundary to be $\theta=2 \pi l / N$, with $l$ an integer.

\subsection{One-pseudoparticle and impurities}

Let us consider the state with one pseudoparticle and one impurity of type $(\omega-1)$, which lies in the sector $r=2 \omega+1$. We seek eigenstates in the form

$$
\Psi_{2 \omega+1}\left(\xi_{1}, \xi_{2}\right)=\sum_{k_{1}<k_{2}}\left\{A_{1}\left(k_{1}, k_{2}\right)\left|\Omega_{1}\left(k_{1}, k_{2}\right)\right\rangle+A_{2}\left(k_{1}, k_{2}\right)\left|\Omega_{2}\left(k_{1}, k_{2}\right)\right\rangle\right\}
$$

We try to build these eigenstates out of translational invariant products of one pseudoparticle excitation with parameter $\xi_{2}$ and one impurity with parameter $\xi_{1}$ :

$$
\Psi_{2 \omega+1}\left(\xi_{1}, \xi_{2}\right)=\left|(\omega-1)\left(\xi_{1}\right)\right\rangle \times \Psi_{2 \omega}\left(\xi_{2}\right)+\Psi_{2 \omega}\left(\xi_{2}\right) \times\left|(\omega-1)\left(\xi_{1}\right)\right\rangle
$$

Using one-pseudopaticle eigenstate solution (3.5) and comparing this with (3.9) we get

$$
\begin{aligned}
& \left|\Omega_{1}\left(k_{1}, k_{2}\right)\right\rangle=\sum_{\alpha=-\omega}^{\omega} \epsilon(\omega) \epsilon(\alpha) q^{-<e_{\omega}+e_{\alpha}, \rho>}\left|k_{1}(\omega-1), k_{2}(-\alpha, \alpha)\right\rangle \\
& \left|\Omega_{2}\left(k_{1}, k_{2}\right)\right\rangle=\sum_{\alpha=-\omega}^{\omega} \epsilon(\omega) \epsilon(\alpha) q^{-<e_{\omega}+e_{\alpha}, \rho>}\left|k_{1}(-\alpha, \alpha), k_{2}(\omega-1)\right\rangle
\end{aligned}
$$

and

$$
A_{1}\left(k_{1}, k_{2}\right)=A_{1} \xi_{1}^{k_{1}} \xi_{2}^{k_{2}} \quad, \quad A_{2}\left(k_{1}, k_{2}\right)=A_{2} \xi_{2}^{k_{1}} \xi_{1}^{k_{2}}
$$

Periodic boundary conditions $A_{1}\left(k_{2}, N+k_{1}\right)=A_{2}\left(k_{1}, k_{2}\right)$ and $A_{i}\left(N+k_{1}, N+k_{2}\right)=A_{i}\left(k_{1}, k_{2}\right), \quad i=1,2$ imply that

$$
A_{1} \xi_{2}^{N}=A_{2} \quad, \quad \xi^{N}=\left(\xi_{1} \xi_{2}\right)^{N}=1
$$

When $\mathcal{H}$ now acts on $\Psi_{2 \omega+1}$, we will get a set of coupled equations for $A_{i}\left(k_{1}, k_{2}\right), i=1,2$. We split the equations into far equations, when the pseudoparticle do not meet the impurity and near equations, containing terms when they are neighbors.

Since the impurity is annihilated by $\mathcal{H}$, the action of $\mathcal{H}$ on $(3.9)$ in the case far (i.e., $\left(k_{2}-k_{1}\right) \geq 3$ ), can be write down directly from (3.7) :

$$
\begin{aligned}
& \left(E_{2 \omega+1}-Q-Q^{-1}\right) A_{1}\left(k_{1}, k_{2}\right)=\epsilon A_{1}\left(k_{1}, k_{2}-1\right)+\epsilon A_{1}\left(k_{1}, k_{2}+1\right) \\
& \left(E_{2 \omega+1}-Q-Q^{-1}\right) A_{2}\left(k_{1}, k_{2}\right)=\epsilon A_{2}\left(k_{1}-1, k_{2}\right)+\epsilon A_{2}\left(k_{1}+1, k_{2}\right)
\end{aligned}
$$


Using the parametrization (3.12), these equations will give us the energy eigenvalues

$$
E_{2 \omega+1}=Q+Q^{-1}+\epsilon\left(\xi_{2}+\xi_{2}^{-1}\right)
$$

To find $\xi_{2}$ we must consider the near equations. First, we compute the action of $\mathcal{H}$ on the coupled near states $\left|\Omega_{1}(k, k+1)\right\rangle$ and $\left|\Omega_{2}(k, k+2)\right\rangle$ :

$$
\begin{array}{r}
\mathcal{H}\left|\Omega_{1}(k, k+1)\right\rangle=\left(Q+Q^{-1}\right)\left|\Omega_{1}(k, k+1)\right\rangle+\epsilon\left|\Omega_{1}(k, k+2)\right\rangle+\epsilon\left|\Omega_{2}(k, k+2)\right\rangle \\
\mathcal{H}\left|\Omega_{2}(k, k+2)\right\rangle=\left(Q+Q^{-1}\right)\left|\Omega_{2}(k, k+2)\right\rangle+\epsilon\left|\Omega_{2}(k-1, k+2)\right\rangle+\epsilon\left|\Omega_{1}(k, k+1)\right\rangle
\end{array}
$$

The last terms in these equations tell us that a pseudoparticle can propagate past the isolated impurity, but in so doing causes a shift in its position by two lattice site. Substituting (3.17) and (3.18) into the eigenvalue equation, we get

$$
\begin{array}{r}
\left(E_{2 \omega+1}-Q-Q^{-1}\right) A_{1}(k, k+1)=\epsilon A_{1}(k, k+2)+\epsilon A_{2}(k, k+2) \\
\left(E_{2 \omega+1}-Q-Q^{-1}\right) A_{2}(k, k+2)=\epsilon A_{2}(k-1, k+2)+\epsilon A_{1}(k, k+1)
\end{array}
$$

These equations, which are not automatically satisfied by the ansatz (3.12), are equivalent to the conditions

$$
A_{1}(k, k) \equiv A_{2}(k, k+2) \quad, \quad A_{1}(k, k+1) \equiv A_{2}(k+1, k+2) .
$$

obtained by subtracting Eq. (3.19) from Eq.(3.14) for $k_{1}=k, k_{2}=k+1$ and by subtracting Eq. (3.20) from Eq.(3.15) for $k_{1}=k, k_{2}=k+2$, respectively. The conditions (3.21) require a modification of the amplitude relation (3.13):

$$
\frac{A_{2}}{A_{1}}=\xi_{1}^{-2}=\xi_{2}^{N} \Rightarrow \xi_{2}^{N} \xi_{1}^{2}=1 \quad \text { or } \quad \xi_{2}^{N-2} \xi^{2}=1
$$

Putting $\xi_{i}=\mathrm{e}^{i \theta_{i}}, i=1,2$, it means $\cos (N-2) \theta_{2}=\cos 2 \theta_{1}$. Hence

$$
\theta_{2}=\frac{2 \pi m \pm 4 \pi l / N}{N-2}, \quad l \text { and } m \text { integers. }
$$

In other words, $\Psi_{2 \omega+1}\left(\xi_{1}, \xi_{2}\right)$ are eigenstates of $\mathcal{H}$ with energy eigenvalues given by $E_{2 \omega+1}=Q+Q^{-1}+$ $2 \epsilon \cos \theta_{2}$. Note that when $N m \pm 2 l$ is a multiple of $(N-2)$ we get states which are degenerate with the one-pseudoparticle states $\Psi_{2 \omega}$, which lie in the sector $r=2 \omega$.

In the sectors $r=2 \omega+l$ we also will find states, which consist of one pseudoparticle with parameter $\xi_{l+1}$ interacting with $l$ impurities, distributing according to (3.1), with parameters $\xi_{i}, i=1,2 \ldots, l$.

The energy of these states is parametrized as in (3.16) and $\xi_{l+1}$ satisfies the condition (3.22) with $\xi=\xi_{1} \cdots \xi_{l} \xi_{l+1}$. It involves only $\xi_{l+1}$ and $\xi_{\text {imp }}=\xi_{1} \xi_{2} \cdots \xi_{l}$, being therefore highly degenerate, i.e.

$$
\xi_{l+1}^{N} \xi_{1}^{2} \xi_{2}^{2} \cdots \xi_{l}^{2}=1
$$

This is to be expected due to the irrelevance of the relative distances, up to jumps of two positions via exchange with a pseudoparticle. Moreover, these results do not depend on impurity type. 


\subsection{Two-pseudoparticles}

The sector $r=4 \omega$ contains, in addition to the cases discussed above, states which consist of two interacting pseudoparticles. We seek eigenstates in the form

$$
\Psi_{4 \omega}\left(\xi_{1}, \xi_{2}\right)=\sum_{k_{1}+1<k_{2}} A\left(k_{1}, k_{2}\right)\left|\Omega\left(k_{1}, k_{2}\right)\right\rangle
$$

Again, we try to build two-pseudoparticle eigenstates out of translational invariant products of onepseudoparticle excitations at $k_{1}$ and $k_{2}\left(k_{2} \geq k_{1}+2\right)$ :

$$
\Psi_{4 \omega}\left(\xi_{1}, \xi_{2}\right)=\Psi_{2 \omega}\left(\xi_{1}\right) \times \Psi_{2 \omega}\left(\xi_{2}\right)+\Psi_{2 \omega}\left(\xi_{2}\right) \times \Psi_{2 \omega}\left(\xi_{1}\right)
$$

Using again (3.5) and comparing (3.26) with (3.25) we get

$$
\left|\Omega\left(k_{1}, k_{2}\right)\right\rangle=\sum_{\alpha, \beta=-\omega}^{\omega} \epsilon(\alpha) \epsilon(\beta) q^{-<2 e_{\omega}+e_{\alpha}+e_{\beta}, \rho>}\left|k_{1}(-\alpha, \alpha), k_{2}(-\beta, \beta)\right\rangle
$$

for $k_{2} \geq k_{1}+3$ and

$$
A\left(k_{1}, k_{2}\right)=A_{12} \xi_{1}^{k_{1}} \xi_{2}^{k_{2}}+A_{21} \xi_{2}^{k_{1}} \xi_{1}^{k_{2}},
$$

for $k_{2} \geq k_{1}+2$.

Periodic boundary conditions $A\left(k_{2}, N+k_{1}\right)=A\left(k_{1}, k_{2}\right)$ and $A\left(N+k_{1}, N+k_{2}\right)=A\left(k_{1}, k_{2}\right)$ imply

$$
A_{12} \xi_{2}^{N}=A_{21} \quad \text { and } \quad \xi^{N}=1
$$

where $\xi=\xi_{1} \xi_{2}\left(\xi_{i}=\mathrm{e}^{i \theta_{i}}, i=1,2\right)$ and the total momentum is $\theta_{1}+\theta_{2}=2 \pi l / N$, with $l$ integer.

Applying $\mathcal{H}$ to the state of (3.25), we obtain a set of equations for the wavefunctions $A\left(k_{1}, k_{2}\right)$. When the two pseudoparticles are separated, $\left(k_{2} \geq k_{1}+3\right)$ these are the following far equations:

$$
\begin{aligned}
\left(E_{4 \omega}-2 Q-2 Q^{-1}\right) A\left(k_{1}, k_{2}\right) & =\epsilon A\left(k_{1}-1, k_{2}\right)+\epsilon A\left(k_{1}+1, k_{2}\right) \\
& +\epsilon A\left(k_{1}, k_{2}-1\right)+\epsilon A\left(k_{1}, k_{2}+1\right)
\end{aligned}
$$

We already know them to be satisfied, if we parametrize $A\left(k_{1}, k_{2}\right)$ by plane waves (3.28). The corresponding energy eigenvalue is

$$
E_{4 \omega}=2 Q+2 Q^{-1}+\epsilon\left(\xi_{1}+\xi_{1}^{-1}+\xi_{2}+\xi_{2}^{-1}\right)
$$

The real problem arises of course, when pseudoparticles are neighbors, so that they interact and we have no guarantee that the total energy is sum of single pseudoparticle energies.

Acting of $\mathcal{H}$ on the state (3.27) gives the following set of equations for the near states

$$
\begin{aligned}
\mathcal{H}|\Omega(k, k+2)\rangle & =2\left(Q+Q^{-1}\right)|\Omega(k, k+2)\rangle+\epsilon|\Omega(k-1, k+2)\rangle \\
& +\epsilon|\Omega(k, k+3)\rangle+U_{k+1}|\Omega(k, k+2)\rangle
\end{aligned}
$$

Before we substitute this result into the eigenvalue equation, we observe that some new states are appearing. In order to incorporate these new states in the eigenvalue problem, we define

$$
U_{k+1}|\Omega(k, k+2)\rangle \equiv \epsilon|\Omega(k, k+1)\rangle+\epsilon|\Omega(k+1, k+2)\rangle
$$


Here we underline that we are using the same notation for these new states. Applying $\mathcal{H}$ to them we obtain

$$
\begin{aligned}
\mathcal{H}|\Omega(k, k+1)\rangle & =\left(Q+Q^{-1}\right)|\Omega(k, k+1)\rangle+\epsilon|\Omega(k-1, k+1)\rangle \\
& +\epsilon|\Omega(k, k+2)\rangle
\end{aligned}
$$

Now, we extend (3.25), the definition of $\Psi_{4 \omega}$, to

$$
\Psi_{4 \omega}\left(\xi_{1}, \xi_{2}\right)=\sum_{k_{1}<k_{2}} A\left(k_{1}, k_{2}\right)\left|\Omega\left(k_{1}, k_{2}\right)\right\rangle
$$

Substituting (3.32) and (3.34) into the eigenvalue equation, we obtain the following set of near equations

$$
\left(E_{4 \omega}-Q-Q^{-1}\right) A(k, k+1)=\epsilon A(k-1, k+1)+\epsilon A(k, k+2)
$$

Using the same parametrization (3.28) for these new wavefunctions, the equation (3.36) gives us the phase shift produced by the interchange of the two interacting pseudoparticles

$$
\frac{A_{21}}{A_{12}}=-\frac{\epsilon(1+\xi)+\left(Q+Q^{-1}\right) \xi_{2}}{\epsilon(1+\xi)+\left(Q+Q^{-1}\right) \xi_{1}}
$$

We thus arrive to the Bethe Ansatz equations which fix the values of $\xi_{1}$ and $\xi_{2}$ in the energy equation (3.31)

$$
\begin{array}{r}
\xi_{2}^{N}=-\frac{1+\xi+\epsilon\left(Q+Q^{-1}\right) \xi_{2}}{1+\xi+\epsilon\left(Q+Q^{-1}\right) \xi_{1}} \\
\xi^{N}=\left(\xi_{1} \xi_{2}\right)^{N}=1
\end{array}
$$

\subsection{Two-pseudoparticles and impurities}

In the sectors $r>6 \omega$, in addition the cases already discussed, we find states with two interacting particles and impurities. Let us now consider two pseudoparticles with one impurity of type $\omega-1$. Theses eigenstates lie in the sector $r=4 \omega+1$ and we seek them in the form

$$
\begin{aligned}
\Psi_{4 \omega+1}\left(\xi_{1}, \xi_{2}, \xi_{3}\right)= & \sum_{k_{1}+1<k_{2}<k_{3}-2} A_{\mathbf{1}}\left(k_{1}, k_{2}, k_{3}\right)\left|\Omega_{1}\left(k_{1}, k_{2}, k_{3}\right)\right\rangle \\
& +\sum_{k_{1}+1<k_{2}<k_{3}} A_{\mathbf{2}}\left(k_{1}, k_{2}, k_{3}\right)\left|\Omega_{2}\left(k_{1}, k_{2}, k_{3}\right)\right\rangle \\
& +\sum_{k_{1}+1<k_{2}<k_{3}-1} A_{\mathbf{3}}\left(k_{1}, k_{2}, k_{3}\right)\left|\Omega_{3}\left(k_{1}, k_{2}, k_{3}\right)\right\rangle
\end{aligned}
$$

In $A_{\mathbf{i}}\left(k_{1}, k_{2}, k_{3}\right)$ the index $\mathbf{i}=1,2,3$ characterizes the impurity position. Comparing (3.39) with the state build from the translational invariant products of two-pseudoparticles with parameters $\xi_{2}$ and $\xi_{3}$ and one-impurity with parameter $\xi_{1}$ :

$$
\begin{aligned}
\Psi_{4 \omega+1}\left(\xi_{1}, \xi_{2}, \xi_{3}\right)= & \left|(\omega-1)\left(\xi_{1}\right)\right\rangle \times \Psi_{2 \omega}\left(\xi_{2}\right) \times \Psi_{2 \omega}\left(\xi_{3}\right) \\
& +\left|(\omega-1)\left(\xi_{1}\right)\right\rangle \times \Psi_{2 \omega}\left(\xi_{3}\right) \times \Psi_{2 \omega}\left(\xi_{2}\right) \\
& +\Psi_{2 \omega}\left(\xi_{2}\right) \times\left|(\omega-1)\left(\xi_{1}\right)\right\rangle \times \Psi_{2 \omega}\left(\xi_{3}\right) \\
& +\Psi_{2 \omega}\left(\xi_{3}\right) \times\left|(\omega-1)\left(\xi_{1}\right)\right\rangle \times \Psi_{2 \omega}\left(\xi_{2}\right) \\
& +\Psi_{2 \omega}\left(\xi_{2}\right) \times \Psi_{2 \omega}\left(\xi_{3}\right) \times\left|(\omega-1)\left(\xi_{1}\right)\right\rangle \\
& +\Psi_{2 \omega}\left(\xi_{3}\right) \times \Psi_{2 \omega}\left(\xi_{2}\right) \times\left|(\omega-1)\left(\xi_{1}\right)\right\rangle
\end{aligned}
$$


we get

$$
\begin{aligned}
\left|\Omega_{1}\left(k_{1}, k_{2}, k_{3}\right)\right\rangle & =\sum_{\alpha, \beta=-\omega}^{\omega} W(\alpha, \beta, \omega)\left|k_{1}(\omega-1), k_{2}(-\alpha, \alpha), k_{3}(-\beta, \beta)\right\rangle \\
\left|\Omega_{2}\left(k_{1}, k_{2}, k_{3}\right)\right\rangle & =\sum_{\alpha, \beta=-\omega}^{\omega} W(\alpha, \beta, \omega)\left|k_{1}(-\alpha, \alpha), k_{2}(\omega-1), k_{3}(-\beta, \beta)\right\rangle \\
\left|\Omega_{3}\left(k_{1}, k_{2}, k_{3}\right)\right\rangle & =\sum_{\alpha, \beta=-\omega}^{\omega} W(\alpha, \beta, \omega)\left|k_{1}(-\alpha, \alpha), k_{2}(-\beta, \beta), k_{3}(\omega-1)\right\rangle
\end{aligned}
$$

where

$$
W(\alpha, \beta, \omega)=\epsilon(\alpha) \epsilon(\beta) q^{-<2 e_{\omega}+e_{\alpha}+e_{\beta}, \rho>}
$$

and the wavefunctions $A_{\mathbf{i}}\left(k_{1}, k_{2}, k_{3}\right)$ which are parametrized by plane waves as

$$
\begin{aligned}
& A_{\mathbf{1}}\left(k_{1}, k_{2}, k_{3}\right)=A_{\mathbf{1} 23} \xi_{1}^{k_{1}} \xi_{2}^{k_{2}} \xi_{3}^{k_{3}}+A_{\mathbf{1 3 2}} \xi_{1}^{k_{1}} \xi_{2}^{k_{3}} \xi_{3}^{k_{2}} \\
& A_{\mathbf{2}}\left(k_{1}, k_{2}, k_{3}\right)=A_{\mathbf{2 1 3}} \xi_{1}^{k_{2}} \xi_{2}^{k_{1}} \xi_{3}^{k_{3}}+A_{\mathbf{2} 31} \xi_{1}^{k_{2}} \xi_{2}^{k_{3}} \xi_{3}^{k_{1}} \\
& A_{\mathbf{3}}\left(k_{1}, k_{2}, k_{3}\right)=A_{\mathbf{3 1 2}} \xi_{1}^{k_{3}} \xi_{2}^{k_{1}} \xi_{3}^{k_{2}}+A_{\mathbf{3} 1} \xi_{1}^{k_{3}} \xi_{2}^{k_{2}} \xi_{3}^{k_{1}} .
\end{aligned}
$$

Periodic boundary conditions read now

$$
\begin{array}{r}
A_{\mathbf{i}}\left(k_{1}, k_{2}, k_{3}\right)=A_{\mathbf{i}}\left(N+k_{1}, N+k_{2}, N+k_{3}\right), \\
A_{\mathbf{i}}\left(k_{2}, k_{3}, N+k_{1}\right)=A_{\mathbf{i}+\mathbf{1}}\left(k_{1}, k_{2}, k_{3}\right), \quad \mathbf{i}=1,2,3 \bmod 3
\end{array}
$$

which imply that

$$
\begin{aligned}
\xi_{1}^{N}=\frac{A_{\mathbf{1 2 3}}}{A_{\mathbf{3} 12}} & =\frac{A_{\mathbf{1 3 2}}}{A_{\mathbf{3} 1}}, \quad \xi_{2}^{N}=\frac{A_{\mathbf{3 1 2}}}{A_{\mathbf{2 3 1}}}=\frac{A_{\mathbf{2 1 3}}}{A_{\mathbf{1 3 2}}}, \\
\xi_{3}^{N} & =\frac{A_{\mathbf{3 2 1}}}{A_{\mathbf{2 1 3}}}=\frac{A_{\mathbf{2 3 1}}}{A_{\mathbf{1 2 3}}}, \quad \xi^{N}=\left(\xi_{1} \xi_{2} \xi_{3}\right)^{N}=1
\end{aligned}
$$

Action of $\mathcal{H}$ on the state $\Psi_{4 \omega+1}$ gives the following set of far equations:

$$
\begin{array}{r}
\left(E_{4 \omega+1}-2 Q-2 Q^{-1}\right) A_{1}\left(k_{1}, k_{2}, k_{3}\right)= \\
+\epsilon A_{1}\left(k_{1}, k_{2}-1, k_{3}\right)+\epsilon A_{1}\left(k_{1}, k_{2}+1, k_{3}\right) \\
+\epsilon A_{1}\left(k_{1}, k_{2}, k_{3}-1\right)+\epsilon A_{1}\left(k_{1}, k_{2}, k_{3}+1\right)
\end{array}
$$

and a similar set of eigenvalue equations for $A_{\mathbf{2}}\left(k_{1}, k_{2}, k_{3}\right)$ and $A_{\mathbf{3}}\left(k_{1}, k_{2}, k_{3}\right)$. The parametrization (3.43) solves these far equations provided that

$$
E_{4 \omega+1}=2 Q+2 Q^{-1}+\epsilon\left(\xi_{2}+\xi_{2}^{-1}+\xi_{3}+\xi_{3}^{-1}\right)
$$

Taking into account the near equations we must split them in three different neighborhood: (i) impurity neighbors of separated pseudoparticles and, (ii) impurity far from neighbors pseudoparticles and (iii) when impurity and pseudoparticles share the same neighborhood.

In the case (i) we consider the second pseudoparticle far and follow the steps for the case of onepseudoparticle with impurity eigenstates. Thus, the near equations can be read off from (3.36)

$$
\begin{array}{r}
\left(E_{4 \omega+1}-2 Q-2 Q^{-1}\right) A_{\mathbf{1}}\left(k, k+1, k_{3}\right)=\epsilon A_{\mathbf{1}}\left(k, k+1, k_{3}-1\right)+\epsilon A_{\mathbf{1}}\left(k, k+1, k_{3}+1\right) \\
+\epsilon A_{\mathbf{1}}\left(k, k+2, k_{3}\right)+\epsilon A_{\mathbf{2}}\left(k, k+2, k_{3}\right)
\end{array}
$$


and a similar set of equations coupling $A_{2}$ and $A_{3}$. It follows from the consistency between $(3.46)$ and (3.48) that

$$
A_{\mathbf{1}}\left(k, k, k_{3}\right) \equiv A_{\mathbf{2}}\left(k, k+2, k_{3}\right)
$$

and similar identification between $A_{2}$ and $A_{3}$. The plane waves $(3.43)$ solve these identifications provided

$$
\xi_{1}^{2}=\frac{A_{123}}{A_{213}}=\frac{A_{132}}{A_{\mathbf{2} 1}} .=\frac{A_{\mathbf{2} 31}}{A_{\mathbf{3} 21}}=\frac{A_{\mathbf{2 1 3}}}{A_{\mathbf{3} 12}}
$$

For the case (ii) we can derive the near equations from those of two-pseudoparticles case. Keeping the impurity far and following the steps $(3.30)-(3.37)$ we get

$$
\left(E_{4 \omega+1}-Q-Q^{-1}\right) A_{1}\left(k_{1}, k, k+1\right)=\epsilon A_{1}\left(k_{1}, k-1, k+1\right)+\epsilon A_{1}\left(k_{1}, k, k+2\right)
$$

and a similar set of equations for $A_{2}$ and $A_{3}$. The case (iii) is obtained from (3.51) for $k_{1}=k-1$.

The parametrization (3.43) solves this provided that

$$
\frac{A_{123}}{A_{132}}=-\frac{\epsilon\left(1+\xi_{3} \xi_{2}\right)+\left(Q+Q^{-1}\right) \xi_{2}}{\epsilon\left(1+\xi_{2} \xi_{3}\right)+\left(Q+Q^{-1}\right) \xi_{3}}
$$

Matching the constraint equations (3.52), (3.50) and (3.45) we arrive to the Bethe equations

$$
\begin{array}{r}
\xi_{a}^{N} \xi_{1}^{2}=-\frac{1+\xi_{b} \xi_{a}+\epsilon\left(Q+Q^{-1}\right) \xi_{a}}{1+\xi_{a} \xi_{b}+\epsilon\left(Q+Q^{-1}\right) \xi_{b}}, \quad a \neq b=2,3 \\
\xi^{N}=\left(\xi_{1} \xi_{2} \xi_{3}\right)^{N}=1, \quad \xi_{1}^{N-4}=1 .
\end{array}
$$

The origin of the exponent $(N-4)$ in the impurity parameter can be understood by saying that after the two pseudoparticles propagate past the impurity, the position of impurity is shifted by four lattice sites.

Next, we can also find eigenstates with two pseudoparticles and more than one impurities. They can be described in the following way: Let us consider an eigenstate with $l>1$ impurities with parameters $\xi_{1}, \xi_{2}, \cdots, \xi_{l}$ and two pseudoparticles with parameters $\xi_{l+1}$ and $\xi_{l+2}$. The energy eigenvalue is

$$
E_{r}=2 Q+2 Q^{-1}+\epsilon\left(\xi_{l+1}+\xi_{l+1}^{-1}+\xi_{l+2}+\xi_{l+2}^{-1}\right)
$$

and the Bethe equations

$$
\begin{array}{r}
\xi_{l+1}^{N} \xi_{1}^{2} \xi_{2}^{2} \cdots \xi_{l}^{2}=-\frac{1+\xi_{l+1} \xi_{l+2}+\epsilon\left(Q+Q^{-1}\right) \xi_{l+1}}{1+\xi_{l+1} \xi_{l+2}+\epsilon\left(Q+Q^{-1}\right) \xi_{l+2}} \\
\xi_{a}^{N-4}=1, \quad a=1,2, \ldots, l
\end{array}
$$

Moreover, $\xi^{N}=1$ with $\xi=\xi_{1} \xi_{2} \cdots \xi_{l+2}$.

\subsection{Three-pseudoparticle eigenstates}

In the sector $r=6 \omega$, in addition to the previously discussed eigenstates of one and two pseudoparticles

with impurities, one can find eigenstates with three interacting pseudoparticles with parameters $\xi_{1}, \xi_{2}$ and $\xi_{3}$. We start seek them in the form

$$
\Psi_{6 \omega}\left(\xi_{1}, \xi_{2}, \xi_{3}\right)=\sum_{k_{1}+2 \leq k_{2} \leq k_{3}-2} A\left(k_{1}, k_{2}, k_{3}\right)\left|\Omega\left(k_{1}, k_{2}, k_{3}\right)\right\rangle
$$


where $\left|\Omega\left(k_{1}, k_{2}, k_{3}\right)\right\rangle=\otimes_{i=1}^{3}\left|\Omega\left(k_{i}\right)\right\rangle$. The corresponding wavefunctions

$$
\begin{aligned}
A\left(k_{1}, k_{2}, k_{3}\right)= & A_{123} \xi_{1}^{k_{1}} \xi_{2}^{k_{2}} \xi_{3}^{k_{3}}+A_{132} \xi_{1}^{k_{1}} \xi_{2}^{k_{3}} \xi_{3}^{k_{2}}+A_{213} \xi_{1}^{k_{2}} \xi_{2}^{k_{1}} \xi_{3}^{k_{3}} \\
& +A_{231} \xi_{1}^{k_{2}} \xi_{2}^{k_{3}} \xi_{3}^{k_{1}}+A_{312} \xi_{1}^{k_{3}} \xi_{2}^{k_{1}} \xi_{3}^{k_{2}}+A_{321} \xi_{1}^{k_{3}} \xi_{2}^{k_{2}} \xi_{3}^{k_{1}}
\end{aligned}
$$

satisfy the periodic boundary conditions

$$
A\left(k_{2}, k_{3}, N+k_{1}\right)=A\left(k_{1}, k_{2}, k_{3}\right), A\left(N+k_{1}, N+k_{2}, N+k_{3}\right)=A\left(k_{1}, k_{2}, k_{3}\right)
$$

which imply that

$$
\begin{aligned}
& \xi_{1}^{N}=\frac{A_{123}}{A_{312}}=\frac{A_{132}}{A_{321}}, \quad \xi_{2}^{N}=\frac{A_{312}}{A_{231}}=\frac{A_{213}}{A_{132}}, \\
& \xi_{3}^{N}=\frac{A_{321}}{A_{213}}=\frac{A_{231}}{A_{123}}, \quad \xi^{N}=\left(\xi_{1} \xi_{2} \xi_{3}\right)^{N}=1
\end{aligned}
$$

These relations show us that the interchange of two-pseudoparticles is independent of the position of the third particle.

Applying $\mathcal{H}$ to (3.57), we obtain a set of equations for $A\left(k_{1}, k_{2}, k_{3}\right)$. When the three pseudoparticles are separated, $\left(k_{1}+2<k_{2}<k_{3}-2\right)$, we get the following far equations:

$$
\begin{aligned}
\left(E_{6 s}-3 Q-3 Q^{-1}\right) A\left(k_{1}, k_{2}, k_{3}\right)= & \epsilon A\left(k_{1}-1, k_{2}, k_{3}\right)+\epsilon A\left(k_{1}+1, k_{2}, k_{3}\right) \\
& +\epsilon A\left(k_{1}, k_{2}-1, k_{3}\right)+\epsilon A\left(k_{1}, k_{2}+1, k_{3}\right) \\
& +\epsilon A\left(k_{1}, k_{2}, k_{3}-1\right)+\epsilon A\left(k_{1}, k_{2}, k_{3}+1\right)
\end{aligned}
$$

It is simple verify that the wavefunctions (3.58) satisfy these far equations provided

$$
E_{6 \omega}=\sum_{n=1}^{3}\left\{Q+Q^{-1}+\xi_{n}+\xi_{n}^{-1}\right\}
$$

Applying $\mathcal{H}$ on the near states we get the following set equations:

$$
\begin{aligned}
\mathcal{H}\left|\Omega\left(k_{1}, k_{1}+2, k_{3}\right)\right\rangle= & \left(2 Q+2 Q^{-1}\right)\left|\Omega\left(k_{1}, k_{1}+2, k_{3}\right)\right\rangle+\epsilon\left|\Omega\left(k_{1}-1, k_{1}+2, k_{3}\right)\right\rangle \\
& +\epsilon\left|\Omega\left(k_{1}, k_{1}+3, k_{3}\right)\right\rangle+\epsilon\left|\Omega\left(k_{1}, k_{1}+2, k_{3}-1\right)\right\rangle \\
& +\epsilon\left|\Omega\left(k_{1}, k_{1}+2, k_{3}+1\right)\right\rangle+U_{k_{1}+1}\left|\Omega\left(k_{1}, k_{1}+2, k_{3}\right)\right\rangle
\end{aligned}
$$

for $k_{3}>k_{1}+4$, which correspond to the meeting of two pseudoparticles at the left of the third pseudoparticle, which is far from of the meeting position.

$$
\begin{aligned}
\mathcal{H}\left|\Omega\left(k_{1}, k_{2}, k_{2}+2\right)\right\rangle= & \left(2 Q+2 Q^{-1}\right)\left|\Omega\left(k_{1}, k_{2}, k_{2}+2\right)\right\rangle+\epsilon\left|\Omega\left(k_{1}-1, k_{2}, k_{2}+2\right)\right\rangle \\
& +\epsilon\left|\Omega\left(k_{1}+1, k_{2}, k_{2}+2\right)\right\rangle+\epsilon\left|\Omega\left(k_{1}, k_{2}-1, k_{2}+2\right)\right\rangle \\
& +\epsilon\left|\Omega\left(k_{1}, k_{2}, k_{2}+3\right)\right\rangle+U_{k_{2}+1}\left|\Omega\left(k_{1}, k_{2}, k_{2}+2\right)\right\rangle
\end{aligned}
$$


for $k_{2}>k_{1}+2$, which correspond to the meeting of two pseudoparticles at the right of the far pseudoparticle. Moreover, there is one set of equations which correspond to the meeting of three pseudoparticles

$$
\begin{aligned}
\mathcal{H}|\Omega(k, k+2, k+4)\rangle= & \left(Q+Q^{-1}\right)|\Omega(k, k+2, k+4)\rangle+\epsilon|\Omega(k-1, k+2, k+4)\rangle \\
& +\epsilon|\Omega(k, k+2, k+5)\rangle+U_{k+1}|\Omega(k, k+2, k+4)\rangle \\
& +U_{k+3}|\Omega(k, k+2, k+4)\rangle
\end{aligned}
$$

In deriving these equations new states made their debut. In order to incorporate these new states in the eigenvalue problem we define:

$$
\begin{aligned}
U_{k_{1}+1}\left|\Omega\left(k_{1}, k_{1}+2, k_{3}\right)\right\rangle & =\epsilon\left|\Omega\left(k_{1}, k_{1}+1, k_{3}\right)\right\rangle+\epsilon\left|\Omega\left(k_{1}+1, k_{1}+2, k_{3}\right)\right\rangle \\
U_{k_{2}+1}\left|\Omega\left(k_{1}, k_{2}, k_{2}+2\right)\right\rangle & =\epsilon\left|\Omega\left(k_{1}, k_{2}+1, k_{2}+2\right)\right\rangle+\epsilon\left|\Omega\left(k_{1}, k_{2}, k_{2}+1\right)\right\rangle \\
U_{k+1}|\Omega(k, k+2, k+4)\rangle & =\epsilon|\Omega(k, k+1, k+4)\rangle+\epsilon|\Omega(k+1, k+2, k+4)\rangle \\
U_{k+3}|\Omega(k, k+2, k+4)\rangle & =\epsilon|\Omega(k, k+3, k+4)\rangle+\epsilon|\Omega(k, k+2, k+3)\rangle
\end{aligned}
$$

Applying $\mathcal{H}$ to these new states the result can be incorporated to the eigenvalue problem provided the definition of $\Psi_{6 \omega}($ (3.57) is extended to

$$
\Psi_{6 \omega}\left(\xi_{1}, \xi_{2}, \xi_{3}\right)=\sum_{k_{1}<k_{2}<k_{3}} A\left(k_{1}, k_{2}, k_{3}\right)\left|\Omega\left(k_{1}, k_{2}, k_{3}\right)\right\rangle
$$

After this we are left with three meeting equations

$$
\begin{gathered}
\left(E_{6 \omega}-2 Q-2 Q^{-1}\right) A\left(k_{1}, k_{1}+1, k_{3}\right)=\epsilon A\left(k_{1}-1, k_{1}+1, k_{3}\right)+\epsilon A\left(k_{1}, k_{1}+2, k_{3}\right) \\
+\epsilon A\left(k_{1}, k_{1}+1, k_{3}-1\right)+\epsilon A\left(k_{1}, k_{1}+1, k_{3}+1\right)
\end{gathered}
$$

for $k_{3}>k_{1}+2$,

$$
\begin{gathered}
\left(E_{6 \omega}-2 Q-2 Q^{-1}\right) A\left(k_{1}, k_{2}, k_{2}+1\right)=\epsilon A\left(k_{1}, k_{2}-1, k_{2}+1\right)+\epsilon A\left(k_{1}, k_{2}, k_{2}+2\right) \\
+\epsilon A\left(k_{1}-1, k_{2}, k_{2}+1\right)+\epsilon A\left(k_{1}+1, k_{2}, k_{2}+1\right)
\end{gathered}
$$

for $k_{1}+2<k_{2}$ and

$$
\left(E_{6 \omega}-Q-Q^{-1}\right) A(k, k+1, k+2)=\epsilon A(k-1, k+1, k+2)+\epsilon A(k, k+1, k+3)
$$

It is easy to verify that the parametrization (3.58) and (3.62) solve these equations provided

$$
\begin{aligned}
& \frac{A_{123}}{A_{213}}=\frac{A_{231}}{A_{321}}=-\frac{1+\xi_{1} \xi_{2}+\epsilon\left(Q+Q^{-1}\right) \xi_{1}}{1+\xi_{1} \xi_{2}+\epsilon\left(Q+Q^{-1}\right) \xi_{2}} \\
& \frac{A_{132}}{A_{231}}=\frac{A_{213}}{A_{312}}=-\frac{1+\xi_{1} \xi_{3}+\epsilon\left(Q+Q^{-1}\right) \xi_{1}}{1+\xi_{1} \xi_{3}+\epsilon\left(Q+Q^{-1}\right) \xi_{3}} \\
& \frac{A_{312}}{A_{321}}=\frac{A_{123}}{A_{132}}=-\frac{1+\xi_{2} \xi_{3}+\epsilon\left(Q+Q^{-1}\right) \xi_{2}}{1+\xi_{2} \xi_{3}+\epsilon\left(Q+Q^{-1}\right) \xi_{3}}
\end{aligned}
$$

Matching these constraints and the periodic boundary conditions (3.60) we get the Bethe Ansatz equations

$$
\begin{aligned}
\xi_{a}^{N} & =\prod_{b \neq a=1}^{3}\left\{-\frac{1+\xi_{a} \xi_{b}+\epsilon\left(Q+Q^{-1}\right) \xi_{a}}{1+\xi_{a} \xi_{b}+\epsilon\left(Q+Q^{-1}\right) \xi_{b}}\right\}, \quad a=1,2,3 \\
\left(\xi_{1} \xi_{2} \xi_{3}\right)^{N} & =1
\end{aligned}
$$




\subsection{General eigenstates}

The generalization to any $r$ is now immediate. Since the Yang-Baxter equations are satisfied, there is only two-pseudoparticle scattering (using the $S$-matrix language). Therefore, neighbor equations, where more then two pseudoparticles become neighbors, are nor expected to give any new restrictions. For instance, in the sector $r=6 \omega$, we saw that the interchange of two-pseudoparticles is independent of the position of the third particle. Thus, in a sector with $p$ pseudoparticles we expect that the $p$-pseudoparticle phase shift will be a sum of $p(p-1) / 2$ two-pseudoparticle phase shift. The energy is given by the sum of single pseudoparticle energies. The corresponding Bethe Ansatz equations depend on the phase shift of two pseudoparticles and on the number of impurity. For a generic sector one can verify that no different neighborhood those discussed above can appear. So, no additional meeting conditions will be encountered. Thus, we can extend the previous results to the $p$-pseudoparticle states in the following way: In a generic sector $r$ with $l$ impurities parametrized by $\xi_{1} \xi_{2} \cdots \xi_{l}$ and $p$ pseudoparticles with parameters $\xi_{l+1} \xi_{l+2} \cdots \xi_{l+p}$, the energy is

$$
E_{r}=\sum_{n=l+1}^{p}\left\{Q+Q^{-1}+\epsilon\left(\xi_{n}+\xi_{n}^{-1}\right)\right\}
$$

with $\xi_{n}$ determined by the Bethe ansatz equations

$$
\begin{aligned}
\xi_{a}^{N} \xi_{1}^{2} \xi_{2}^{2} \cdots \xi_{l}^{2} & =\prod_{b \neq a=l+1}^{l+p}\left\{-\frac{1+\xi_{b} \xi_{a}+\epsilon\left(Q+Q^{-1}\right) \xi_{a}}{1+\xi_{a} \xi_{b}+\epsilon\left(Q+Q^{-1}\right) \xi_{b}}\right\} \\
\xi_{c}^{N-2 p} & =1, \quad c=1,2, \ldots, l \\
\xi^{N} & =1, \quad \xi=\xi_{1} \xi_{2} \cdots \xi_{l} \xi_{l+1} \xi_{l+2} \cdots \xi_{l+p} .
\end{aligned}
$$

The energy eigenvalues and the Bethe equations depend on the deformation parameter $q$, through the relation (2.1):

$$
Q+Q^{-1}=\left\{\begin{array}{lll}
{[2 s+1]} & \text { for } \quad A_{1} \\
{[2 n-1][n+1 / 2] /[n-1 / 2]} & \text { for } \quad B_{n}(n \geq 2) \\
{[n][2 n+2] /[n+1]} & \text { for } \quad C_{n}(n \geq 1) \\
{[n][2 n-2] /[n-1]} & \text { for } \quad D_{n}(n \geq 3)
\end{array}\right.
$$

We obtained thus the spectra with periodic boundary conditions of quantum spin-chain models, arising as representations of the Temperley-Lieb algebra. As expected, all these models have equivalent spectra up to degeneracies of their eigenvalues. From a suitable sorting of the parameters $\xi_{i}$, one can insure that the spectra of lower- $r$ sectors are contained entirely in the higher- $r$ sectors.

\section{Bethe Ansatz: Non-local boundary conditions}

It is the purpose of this section to present and solve, via coordinate Bethe ansatz, the quantum group invariant closed TL Hamiltonians which can be written as [19]:

$$
\mathcal{H}=\sum_{k=1}^{N-1} U_{k}+\mathcal{U}_{0}
$$


where $U_{k}$ is a Temperley-Lieb operator and bt $=\mathcal{U}_{0}$ is non-local term defined through of a operator $G$ which plays the role of the translation operator

$$
\mathcal{U}_{0}=G U_{N-1} G^{-1} \quad, \quad G=\left(Q-U_{1}\right)\left(Q-U_{2}\right) \cdots\left(Q-U_{N-1}\right)
$$

satisfying $[\mathcal{H}, G]=0$ and additionally invariance with respect to the quantum algebra. The operator $G$ shifts the $U_{k}$ by one unit $G U_{k} G^{-1}=U_{k+1}$ and maps $\mathcal{U}_{0}$ into $U_{1}$, which manifest the translational invariance of $\mathcal{H}$. In this sense the Hamiltonian (4.1) is periodic.

For the $q$-deformed $A$-D Temperley-Lieb algebra, the matrix elements of $U_{k}$ is again given by (2.8), i.e. :

$$
\begin{array}{r}
\langle\nu, \lambda|U| \mu, \kappa\rangle=\epsilon(\mu) \epsilon(\nu) q^{-<e_{\mu}+e_{\nu}, \rho>} \delta_{\mu+\kappa, 0} \delta_{\nu+\lambda, 0} \\
\mu, \nu, \kappa, \lambda \in J
\end{array}
$$

From (4.3) we choose an particular Bethe state

$$
|\Omega(k)\rangle=\sum_{\alpha=-\omega}^{\omega} \epsilon(\omega) \epsilon(\alpha) q^{-<e_{\omega}+e_{\alpha}, \rho>}|k(-\alpha, \alpha)\rangle
$$

which is an eigenstate of $U_{k}$ and it is shifted by one unit under the action of $U_{k \pm 1}$

$$
\begin{array}{r}
U_{k}|\Omega(k)\rangle=\left(Q+Q^{-1}\right)|\Omega(k)\rangle \\
U_{k \pm 1}|\Omega(k)\rangle=\epsilon|\Omega(k \pm 1)\rangle \quad, \quad U_{k}|\Omega(k \pm)\rangle=\epsilon|\Omega(k)\rangle \\
U_{k}|\Omega(l)\rangle=0 \quad \text { for } \quad k \neq\{l-1, l, l+1\}
\end{array}
$$

where $\epsilon=1$ for $B_{n}, D_{n}$ and $A_{1}$ ( $s$ integer) and $\epsilon=-1$ for $C_{n}$ and $A_{1}$ ( $s$ semi-integer).

The action of the operator $G$ on the states $|\Omega(k)\rangle$ can be easily computed using (4.5): It is simple on the bulk and at the left boundary

$$
G|\Omega(k)\rangle=-\epsilon Q^{N-2}|\Omega(k+1)\rangle \quad, \quad 1 \leq k \leq N-2
$$

but manifests its nonlocality at the right boundary

$$
G|\Omega(N-1)\rangle=\epsilon Q^{N-2} \sum_{k=1}^{N-1}(-\epsilon Q)^{-k}|\Omega(N-k)\rangle
$$

Similarly, the action of the operator $G^{-1}=\left(Q^{-1}-U_{N-1}\right) \cdots\left(Q^{-1}-U_{1}\right)$ is simple on the bulk and at the right boundary

$$
G^{-1}|\Omega(k)\rangle=-\epsilon Q^{-N+2}|\Omega(k-1)\rangle \quad, \quad 2 \leq k \leq N-1
$$

and non-local at the left boundary

$$
G^{-1}|\Omega(1)\rangle=\epsilon Q^{-N+2} \sum_{k=1}^{N-1}(-\epsilon Q)^{k}|\Omega(k)\rangle .
$$

Now we proceed the diagonalization of $\mathcal{H}$ as was made for the periodic case. As (4.1) and (1.2) have the same bulk, i.e., differences came from the boundary terms, we will keep all results relating to the bulk of the periodic case presented in the previous section. 


\subsection{One-pseudoparticle eigenstates}

Let us consider one free pseudoparticle which lies in the sector $r=2 \omega$

$$
\Psi_{2 \omega}=\sum_{k=1}^{N-1} A(k)|\Omega(k)\rangle .
$$

The action of the operator $\mathcal{U}=\sum_{k=1}^{N-1} U_{k}$ on the states $|\Omega(k)\rangle$ can be computed using (4.5):

$$
\begin{array}{r}
\mathcal{U}|\Omega(1)\rangle=\left(Q+Q^{-1}\right)|\Omega(1)\rangle+\epsilon|\Omega(2)\rangle \\
\mathcal{U}|\Omega(k)\rangle=\left(Q+Q^{-1}\right)|\Omega(k)\rangle+\epsilon|\Omega(k-1)\rangle+\epsilon|\Omega(k+1)\rangle \\
\text { for } 2 \leq k \leq N-2 \\
\mathcal{U}|\Omega(N-1)\rangle=\left(Q+Q^{-1}\right)|\Omega(N-1)\rangle+\epsilon|\Omega(N-2)\rangle .
\end{array}
$$

and using (4.6)-(4.9) one can see that the action of $\mathcal{U}_{0}=G U_{N-1} G^{-1}$ vanishes on the bulk

$$
\mathcal{U}_{0}|\Omega(k)\rangle=0 \quad, \quad 2 \leq k \leq N-2
$$

and is nonlocal at the boundaries

$$
\mathcal{U}_{0}|\Omega(1)\rangle=-\epsilon \sum_{k=1}^{N-1}(-\epsilon Q)^{k}|\Omega(k)\rangle, \quad \mathcal{U}_{0}|\Omega(N-1)\rangle=-\epsilon \sum_{k=1}^{N-1}(-\epsilon Q)^{-N+k}|\Omega(k)\rangle .
$$

which are connected by

$$
\mathcal{U}_{0}|\Omega(N-1)\rangle=(-\epsilon Q)^{-N} \mathcal{U}_{0}|\Omega(1)\rangle .
$$

From these equations we can understood the role of $\mathcal{U}_{0}$ : Although the Hamiltonian (4.1) is a global operator, it manifests the property of essential locality. From the physical point of view, this type of models exhibit behavior similar to closed chains with twisted boundary conditions.

Before we substitute these results into the eigenvalue equation, we will define two new states

$$
\epsilon|\Omega(0)\rangle=\mathcal{U}_{0}|\Omega(1)\rangle, \quad \epsilon|\Omega(N)\rangle=\mathcal{U}_{0}|\Omega(N-1)\rangle
$$

to include the cases $k=0$ and $k=N$ into the definition of $\Psi_{2 \omega}$, equation (4.10). Finally, the action of $\mathcal{H}=\mathcal{U}+\mathcal{U}_{0}$ on the states $|\Omega(k)\rangle$ is

$$
\begin{array}{r}
\mathcal{H}|\Omega(0)\rangle=\left(Q+Q^{-1}\right)|\Omega(0)\rangle+(-\epsilon Q)^{N} \epsilon|\Omega(N-1)\rangle+\epsilon|\Omega(1)\rangle \\
\mathcal{H}|\Omega(k)\rangle=\left(Q+Q^{-1}\right)|\Omega(k)\rangle+\epsilon|\Omega(k-1)\rangle+\epsilon|\Omega(k+1)\rangle \\
\text { for } 1 \leq k \leq N-2 \\
\mathcal{H}|\Omega(N-1)\rangle=\left(Q+Q^{-1}\right)|\Omega(N-1)\rangle+\epsilon|\Omega(N-2)\rangle+(-\epsilon Q)^{-N} \epsilon|\Omega(0)\rangle \\
\mathcal{H}|\Omega(N)\rangle=\left(Q+Q^{-1}\right)|\Omega(N)\rangle+\epsilon|\Omega(N-1)\rangle+(-\epsilon Q)^{-N} \epsilon|\Omega(1)\rangle
\end{array}
$$


Substituting these results into the eigenvalue equation $\mathcal{H} \Psi_{2 \omega}=E_{2 \omega} \Psi_{2 \omega}$ we get a complete set of eigenvalue equations for the wavefunctions

$$
\begin{array}{r}
E_{2 s} A(k)=\left(Q+Q^{-1}\right) A(k)+\epsilon A(k-1)+\epsilon A(k+1) \\
\text { for } 1 \leq k \leq N-1
\end{array}
$$

provided the following boundary conditions

$$
(-\epsilon Q)^{N} A(k)=A(N+k)
$$

are satisfied.

The plane wave parametrization $A(k)=A \xi^{k}$ solves these eigenvalue equations and the boundary conditions provided that:

$$
\begin{array}{r}
E_{2 \omega}=Q+Q^{-1}+\epsilon\left(\xi+\xi^{-1}\right) \\
\xi^{N}=(-\epsilon Q)^{N}
\end{array}
$$

where $\xi=\mathrm{e}^{i \theta}$ and $\theta$ being the momentum.

\subsection{Two-pseudoparticle eigenstates}

Let us now consider the sector $r=4 \omega$, where we can find an eigenstate with two interacting pseudoparticles. We seek the corresponding eigenfunction as products of single pseudoparticles eigenfunctions, i.e.

$$
\Psi_{4 \omega}=\sum_{k_{1}+1<k_{2}} A\left(k_{1}, k_{2}\right)\left|\Omega\left(k_{1}, k_{2}\right)\right\rangle
$$

where

$$
\left|\Omega\left(k_{1}, k_{2}\right)\right\rangle=\sum_{\alpha, \beta=-\omega}^{\omega} \epsilon(\alpha) \epsilon(\beta) q^{-<2 e_{\omega}+e_{\alpha}+e_{\beta}, \rho>}\left|k_{1}(-\alpha, \alpha), k_{2}(-\beta, \beta)\right\rangle
$$

To solve the eigenvalue equation $\mathcal{H} \Psi_{4 \omega}=E_{4 \omega} \Psi_{4 \omega}$, we recall (4.5) to get the action of $\mathcal{U}$ and $\mathcal{U}_{0}$ on the states $\left|\Omega\left(k_{1}, k_{2}\right)\right\rangle$. Here we have to consider four cases: (i) when the two pseudoparticles are separated in the bulk, the action of $\mathcal{U}$ is

$$
\begin{aligned}
\mathcal{U}\left|\Omega\left(k_{1}, k_{2}\right)\right\rangle=2\left(Q+Q^{-1}\right)\left|\Omega\left(k_{1}, k_{2}\right)\right\rangle & +\epsilon\left|\Omega\left(k_{1}-1, k_{2}\right)\right\rangle+\epsilon\left|\Omega\left(k_{1}+1, k_{2}\right)\right\rangle \\
& +\epsilon\left|\Omega\left(k_{1}, k_{2}-1\right)\right\rangle+\epsilon\left|\Omega\left(k_{1}, k_{2}+1\right)\right\rangle
\end{aligned}
$$

i.e., for $k_{1} \geq 2$ and $k_{1}+3 \leq k_{2} \leq N-2$; (ii) when the two pseudoparticles are separated but one of them or both are at the boundaries

$$
\begin{aligned}
& \mathcal{U}\left|\Omega\left(1, k_{2}\right)\right\rangle=2\left(Q+Q^{-1}\right)\left|\Omega\left(1, k_{2}\right)\right\rangle+\epsilon\left|\Omega\left(2, k_{2}\right)\right\rangle+\epsilon\left|\Omega\left(1, k_{2}-1\right)\right\rangle \\
& +\epsilon\left|\Omega\left(1, k_{2}+1\right)\right\rangle \\
& \mathcal{U}\left|\Omega\left(k_{1}, N-1\right)\right\rangle=2\left(Q+Q^{-1}\right)\left|\Omega\left(k_{1}, N-1\right)\right\rangle+\epsilon\left|\Omega\left(k_{1}-1, N-1\right)\right\rangle \\
& +\epsilon\left|\Omega\left(k_{1}+1, N-1\right)\right\rangle+\epsilon\left|\Omega\left(k_{1}, N-2\right)\right\rangle
\end{aligned}
$$




$$
\mathcal{U}|\Omega(1, N-1)\rangle=2\left(Q+Q^{-1}\right)|\Omega(1, N-1)\rangle+\epsilon|\Omega(2, N-1)\rangle+\epsilon|\Omega(1, N-2)\rangle
$$

where $2 \leq k_{1} \leq N-4$ and $4 \leq k_{2} \leq N-2$; (iii) when the two pseudoparticles are neighbors in the bulk

$$
\begin{array}{r}
\mathcal{U}|\Omega(k, k+2)\rangle=2\left(Q+Q^{-1}\right)|\Omega(k, k+2)\rangle+\epsilon|\Omega(k-1, k+2)\rangle+\epsilon|\Omega(k, k+3)\rangle \\
+U_{k+1}|\Omega(k, k+2)\rangle
\end{array}
$$

for $2 \leq k \leq N-4$ and (iv) when the two pseudoparticles are neighbors and at the boundaries

$$
\begin{array}{r}
\mathcal{U}|\Omega(1,3)\rangle=2\left(Q+Q^{-1}\right)|\Omega(1,3)\rangle+\epsilon|\Omega(1,4)\rangle+U_{2}|\Omega(1,3)\rangle \\
\mathcal{U}|\Omega(N-3, N-1)\rangle=2\left(Q+Q^{-1}\right)|\Omega(N-3, N-1)\rangle+\epsilon|\Omega(N-4, N-1)\rangle \\
+U_{N-2}|\Omega(N-3, N-1)\rangle
\end{array}
$$

Moreover, the action of $\mathcal{U}_{0}$ does not depend on the pseudoparticles are neither separated nor neighbors. It is vanishes in the bulk

$$
\mathcal{U}_{0}\left|\Omega\left(k_{1}, k_{2}\right)\right\rangle=0 \quad \text { for } \quad k_{1} \neq 1 \text { and } k_{2} \neq N-1,
$$

and different of zero at the boundaries:

$$
\begin{aligned}
\mathcal{U}_{0}\left|\Omega\left(1, k_{2}\right)\right\rangle= & -\epsilon \sum_{k=1}^{k_{2}-2}(-\epsilon Q)^{k}\left|\Omega\left(k, k_{2}\right)\right\rangle-(-\epsilon Q)^{k_{2}-1} U_{k_{2}}\left|\Omega\left(k_{2}-1, k_{2}+1\right)\right\rangle \\
& -\epsilon \sum_{k=k_{2}+2}^{N-1}(-\epsilon Q)^{k-2}\left|\Omega\left(k_{2}, k\right)\right\rangle \\
& \mathcal{U}_{0}\left|\Omega\left(k_{1}, N-1\right)\right\rangle=(-\epsilon Q)^{-N+2} \mathcal{U}_{0}\left|\Omega\left(1, k_{2}\right)\right\rangle
\end{aligned}
$$

where $2 \leq k_{1} \leq N-3$ and $3 \leq k_{2} \leq N-2$.

Following the same procedure of one-pseudoparticle case we again define new states in order to have consistency between bulk and boundaries terms

$$
\begin{aligned}
\mathcal{U}_{0}\left|\Omega\left(1, k_{2}\right)\right\rangle & =\epsilon\left|\Omega\left(0, k_{2}\right)\right\rangle, \quad \mathcal{U}_{0}\left|\Omega\left(k_{1}, N-1\right)\right\rangle=\epsilon\left|\Omega\left(k_{1}, N\right)\right\rangle \\
\mathcal{U}_{0}|\Omega(1, N-1)\rangle & =\epsilon|\Omega(0, N-1)\rangle+\epsilon|\Omega(1, N)\rangle \\
U_{k+1}|\Omega(k, k+2)\rangle & =\epsilon|\Omega(k, k+1)\rangle+\epsilon|\Omega(k+1, k+2)\rangle
\end{aligned}
$$

Acting with $\mathcal{H}$ on these new states, we get

$$
\begin{aligned}
\mathcal{H}\left|\Omega\left(0, k_{2}\right)\right\rangle= & 2\left(Q+Q^{-1}\right)\left|\Omega\left(0, k_{2}\right)\right\rangle+\epsilon\left|\Omega\left(0, k_{2}-1\right)\right\rangle+\epsilon\left|\Omega\left(0, k_{2}+1\right)\right\rangle \\
& +\epsilon\left|\Omega\left(1, k_{2}\right)\right\rangle+(-\epsilon Q)^{N-2} \epsilon\left|\Omega\left(k_{2}, N-1\right)\right\rangle \\
\mathcal{H}\left|\Omega\left(k_{1}, N\right)\right\rangle= & 2\left(Q+Q^{-1}\right)\left|\Omega\left(k_{1}, N\right)\right\rangle+\epsilon\left|\Omega\left(k_{1}-1, N\right)\right\rangle+\epsilon\left|\Omega\left(k_{1}+1, N\right)\right\rangle \\
& +\epsilon\left|\Omega\left(k_{1}, N-1\right)\right\rangle+(-\epsilon Q)^{-N+2} \epsilon\left|\Omega\left(1, k_{1}\right)\right\rangle \\
\mathcal{H} \mid \Omega(k, k+1\rangle= & \left(Q+Q^{-1}\right)|\Omega(k, k+1\rangle+\epsilon| \Omega(k-1, k+1\rangle+\epsilon \mid \Omega(k, k+2\rangle
\end{aligned}
$$


Substituting these results into the eigenvalue equation, we get the following equations for wavefunctions corresponding to the separated pseudoparticles.

$$
\begin{aligned}
\left(E_{4 \omega}-2 Q-2 Q^{-1}\right) A\left(k_{1}, k_{2}\right)= & \epsilon A\left(k_{1}-1, k_{2}\right)+\epsilon A\left(k_{1}+1, k_{2}\right) \\
& +\epsilon A\left(k_{1}, k_{2}-1\right)+\epsilon A\left(k_{1}, k_{2}+1\right)
\end{aligned}
$$

i.e., for $k_{1} \geq 1$ and $k_{1}+3 \leq k_{2} \leq N-1$. The boundary conditions read now

$$
A\left(k_{2}, N+k_{1}\right)=(-\epsilon Q)^{N-2} A\left(k_{1}, k_{2}\right) .
$$

The parametrization for the wavefunctions

$$
A\left(k_{1}, k_{2}\right)=A_{12} \xi_{1}^{k_{1}} \xi_{2}^{k_{2}}+A_{21} \xi_{1}^{k_{2}} \xi_{2}^{k_{1}}
$$

solves the equation (4.36) provided that

$$
E_{4 s}=2\left(Q+Q^{-1}\right)+\epsilon\left(\xi_{1}+\xi_{1}^{-1}+\xi_{2}+\xi_{2}^{-1}\right)
$$

and the boundary conditions (4.37) provided that

$$
\xi_{2}^{N}=(-\epsilon Q)^{N-2} \frac{A_{21}}{A_{12}} \quad, \quad \xi_{1}^{N}=(-\epsilon Q)^{N-2} \frac{A_{12}}{A_{21}} \Rightarrow \xi^{N}=(-\epsilon Q)^{2(N-2)}
$$

where $\xi=\xi_{1} \xi_{2}=e^{i\left(\theta_{1}+\theta_{2}\right)}, \theta_{1}+\theta_{2}$ being the total momenta.

Now we include the new states (4.32) into the definition of $\Psi_{4 \omega}$ in order to extend (4.20) to

$$
\Psi_{4 \omega}=\sum_{k_{1}<k_{2}} A\left(k_{1}, k_{2}\right) \mid \Omega\left(k_{1}, k_{2}\right\rangle .
$$

Here we have used the same notation for separated and neighboring states.

Substituting (4.26) and (4.35) into the eigenvalue equation, we get

$$
\left(E_{4 \omega}-Q-Q^{-1}\right) A(k, k+1)=\epsilon A(k-1, k+1)+\epsilon A(k, k+2)
$$

which gives us the phase shift produced by the interchange of the two pseudoparticles

$$
\frac{A_{21}}{A_{12}}=-\frac{1+\xi+\epsilon\left(Q+Q^{-1}\right) \xi_{2}}{1+\xi+\epsilon\left(Q+Q^{-1}\right) \xi_{1}} .
$$

We thus arrive to the Bethe ansatz equations which fix the values of $\xi_{1}$ and $\xi_{2}$ :

$$
\begin{aligned}
\xi_{2}^{N} & =(-\epsilon Q)^{N-2}\left\{-\frac{1+\xi+\epsilon\left(Q+Q^{-1}\right) \xi_{2}}{1+\xi+\epsilon\left(Q+Q^{-1}\right) \xi_{1}}\right\}, \\
\xi_{1}^{N} \xi_{2}^{N} & =(-\epsilon Q)^{2(N-2)}
\end{aligned}
$$




\subsection{General eigenstates}

Thus in the sector $r=2 \omega p$, we expect that the $p$-pseudoparticle phase shift will be a sum of twopseudoparticle phase shifts and the energy is given by

$$
E_{p(2 s)}=\sum_{n=1}^{p}\left\{Q+Q^{-1}+\epsilon\left(\xi_{n}+\xi_{n}^{-1}\right)\right\}
$$

where

$$
\begin{gathered}
\xi_{a}^{N}=\left(-\epsilon_{s} Q\right)^{N-2 p+2} \prod_{b \neq a}^{p}\left\{-\frac{1+\xi_{a} \xi_{b}+\epsilon\left(Q+Q^{-1}\right) \xi_{a}}{1+\xi_{a} \xi_{b}+\epsilon\left(Q+Q^{-1}\right) \xi_{b}}\right\}, \quad a=1, \ldots, p \\
\left(\xi_{1} \xi_{2} \cdots \xi_{p}\right)^{N}=(-\epsilon Q)^{p(N-2 p+2)}
\end{gathered}
$$

The corresponding eigenstates are

$$
\Psi_{r}\left(\xi_{1}, \xi_{2}, \ldots \xi_{p}\right)=\sum_{1 \leq k_{1}<\ldots<k_{p} \leq N-1} A\left(k_{1}, k_{2}, \ldots, k_{p}\right)\left|\Omega\left(k_{1}, k_{2}, \ldots, k_{p}\right)\right\rangle
$$

where $\left|\Omega\left(k_{1}, k_{2}, \ldots, k_{p}\right)\right\rangle=\otimes_{i=1}^{p}\left|\Omega\left(k_{i}\right)\right\rangle$ and the wavefunctions satisfy the following boundary conditions

$$
A\left(k_{1}, k_{2}, \ldots, k_{p}, N+k_{1}\right)=(-\epsilon Q)^{N-2 p+2} A\left(k_{1}, k_{2}, \ldots, k_{p}\right)
$$

It is not all, in a sector $r$ we may have $p$ pseudoparticle and $N_{\omega-1}, N_{\omega-2}, \ldots, N_{-\omega+1}$ impurities of the type $(\omega-1),(\omega-2), \ldots,(-\omega+1)$, respectively, such that

$$
N_{\omega-1}+2 N_{\omega-2}+\cdots+(2 \omega-1) N_{-\omega+1}=r-2 \omega p
$$

We called impurity a state $|\alpha, k\rangle$ flanked by at least two states $|\beta, k \pm 1\rangle$ such that $\alpha+\beta \neq 0$. Since $\mathcal{H}$ is a sum of projectors on spin zero, these states are also annihilated by $\mathcal{U}_{0}$. Therefore the impurities play here the same role as in the periodic case. It means that for a sector $r$ with $l$ impurities with parameters $\xi_{1}, \ldots, \xi_{l}$ and $p$ pseudoparticles with parameters $\xi_{l+1}, \ldots, \xi_{l+p}$ the energy is given by (4.46), and the Bethe equations do not depend on impurity type and are given by

$$
\xi_{a}^{N} \xi_{1}^{2} \xi_{2}^{2} \cdots \xi_{l}^{2}=(-\epsilon Q)^{N-2 p+2} \prod_{b \neq a=l+1}^{l+p}\left\{-\frac{1+\xi_{a} \xi_{b}+\epsilon\left(Q+Q^{-1}\right) \xi_{a}}{1+\xi_{a} \xi_{b}+\epsilon\left(Q+Q^{-1}\right) \xi_{b}}\right\}
$$

with $a=l+1, l+2, \ldots, l+p \quad, \quad p \geq 1$, and

$$
\xi^{2 p}\left(\xi_{l+1} \cdots \xi_{l+p}\right)^{N-2 p}=(-\epsilon Q)^{p(N-2 p+2)}
$$

where $\xi=\xi_{1} \xi_{2} \cdots \xi_{l} \xi_{l+1} \cdots \xi_{l+p}$.

We have shown that these closed Temperley-Lieb quantum invariant spin chains can be solved by the coordinate Bethe ansatz. A consequence of the nonlocal terms $\mathcal{U}_{0}$ is the arising of boundary conditions depending on the quantum group parameter $q$ via the relation $Q+Q^{-1}=\operatorname{Tr}_{V_{\Lambda}}\left(q^{-2 \rho}\right)$. It is also $p$ pseudoparticle dependent (which is equal to spin sector $r$ for $A_{1}$, when $s=1 / 2$ ).

For the algebra $A_{1}$, and $s=1 / 2, \mathrm{Q}=\mathrm{q}$ and $U_{k}$ are $4 \times 4$ matrices giving a nearest-neighbour interaction $U_{k}=q-\sigma_{k}^{+} \sigma_{k+1}^{-}-\sigma_{k}^{-} \sigma_{k+1}^{+}-\left(q+q^{-1}\right) / 4\left(\sigma_{k}^{z} \sigma_{k+1}^{z}+1\right)+\left(q+q^{-1}\right) / 4\left(\sigma_{k}^{z}-\sigma_{k+1}^{z}-2\right)$. This Hamiltonian was investigated in Ref. [20] and we summarize some basic results regarding this case. Assuming $\mathrm{q}=\exp (i \varphi)$ 
some interesting properties were found. For instance, the spin $L$ of the ground state becomes $\varphi$ dependent. For any $\mathrm{N}$ (even), $L$ depends on the value of $\varphi$ according to :

$$
\begin{array}{rlrlrl}
L & =0 & & \text { for } & \frac{\pi}{2} & <\varphi<\pi \\
L & =l & & \text { for } \frac{\pi}{2(l+1)} & <\varphi<\frac{\pi}{2 l} \\
L & =\frac{N}{2} & & \text { for } & 0 & <\varphi<\frac{\pi}{N}
\end{array}
$$

The ground state is non-degenerate (up to the trivial $\mathrm{SU}_{q}$ degeneracy). At the edges of the intervals, $\varphi=\pi / 2 l$, additional degeneracies occur. These transitions to higher spins resemble the incommensurate transition obtained in various other models.

From the statistical mechanics point of view the Hamiltonian presents critical behavior and it is conformal invariant. The central charge (or conformal anomaly) is :

$$
c=1-\frac{6(\pi-\varphi)^{2}}{\pi \varphi}, \quad \varphi \in[\pi / 2, \pi] .
$$

In particular, if we choose the rational form :

$$
\varphi=\frac{\pi m}{(m+1)}, \quad m=3,4 \ldots
$$

then

$$
c=1-\frac{6}{m(m+1)},
$$

which give us the conformal anomalies of the minimal unitary models.

The connection between the Hamiltonian of the closed $\mathrm{SU}_{q}(2)$ invariant chain and the unitary minimal series was explored in [35]. For a generic irrational $\varphi$ one can decompose the space of states into the direct sum of irreducible representations of the quantum group which are in one-to-one correspondence with the usual SU(2) representations.

We have constructed and diagonalized numerically the Hamiltonian for small values of $\mathrm{N}$ and $\mathrm{s}=1 / 2$, 1. We checked that, for a given $N, s=1 / 2$ and 1 have the same spectra up to degeneracies.

\section{$5 \quad$ Bethe Ansatz: Free boundary conditions}

It is for free boundary conditions that the Hamiltonian (2.7) naturally commutes with the quantum group $\mathcal{U}_{q}\left(X_{n}\right)$. Since the our linear combination (3.2) left all models with the same status, which concern to the coordinate Bethe ansatz, we expect that all procedure developed for the coordinate Bethe ansatz with free boundary conditions in [6] for the case $A_{1}(s=1 / 2)$. can be used here. To show this we recall the previous section, taking into account $\mathcal{U}_{0}=0$, where almost all equations can be seized for the free boundary conditions eigenvalue problem. 


\subsection{One-pseudoparticle}

In this sector, the eigenstate is given by (4.10):

$$
\Psi_{2 \omega}(\xi)=\sum_{k=1}^{N-1} A(k)|\Omega(k)\rangle
$$

where $|\Omega(k)\rangle$ is again given by $(4.4)$.

The action of $\mathcal{H}$ on the states $|\Omega(k)\rangle$ is given by (4.11), which gives us the following eigenvalue equations

$$
\left(E_{2 \omega}-Q-Q^{-1}\right) A(k)=\epsilon A(k-1)+\epsilon A(k+1), \quad 2 \leq k \leq N-2
$$

At the boundaries, we get more two slightly different equations

$$
\begin{array}{r}
\left(E_{2 \omega}-Q-Q^{-1}\right) A(1)=\epsilon A(2) \\
\left(E_{2 \omega}-Q-Q^{-1}\right) A(N-1)=\epsilon A(N-2)
\end{array}
$$

where $\epsilon=1$ for $B_{n}, D_{n}$ and $A_{1}$ ( $s$ integer) and $\epsilon=-1$ for $C_{n}$ and $A_{1}$ ( $s$ semi-integer). We now try as a solution

$$
A(k)=\mathrm{A}(\theta) \xi^{k}-\mathrm{A}(-\theta) \xi^{-k}
$$

where $\xi=e^{i \theta}, \theta$ being the momenta. Substituting this in equation (5.2) we obtain the energy eigenvalue associated with a free pseudoparticle with free boundary conditions

$$
E_{2 \omega}=Q+Q^{-1}+\epsilon\left(\xi+\xi^{-1}\right)
$$

We want equations (5.2) to be valid for $k=1$ and $k=N-1$ also, where $A(0)$ and $A(N)$ are defined by (5.4). Matching (5.2) and (5.3) we get the end conditions

$$
A(0)=0 \quad \text { and } \quad A(N)=0
$$

implying that $A(\theta)=A(-\theta)$ and $\xi^{2 N}=1$, respectively. $A(\theta)$ it now determined ( up to a factor that is invariant under $\theta \longleftrightarrow-\theta)$, to be equal to $\xi^{-N}$.

\subsection{One pseudoparticle and impurities}

Differently from the previous cases, due to the lack of periodicity, the impurity positions are fixed. So, they have a different role in the eigenvalue problem with free boundary conditions. For instance, let us consider the case of one impurity of the type $\omega-1$, with parameter $\xi_{1}$ and one pseudoparticle with parameter $\xi_{2}$. This eigenstate lies in the sector $r=2 \omega+1$ and we can write

$$
\Psi_{2 \omega+1}\left(\xi_{1}, \xi_{2}\right)=\sum_{k_{1}<k_{2}}\left\{A_{1}\left(k_{1}, k_{2}\right)\left|\Omega_{1}\left(k_{1}, k_{2}\right)\right\rangle+A_{2}\left(k_{1}, k_{2}\right)\left|\Omega_{2}\left(k_{1}, k_{2}\right)\right\rangle\right\}
$$

where $\left|\Omega_{i}\left(k_{1}, k_{2}\right)\right\rangle, i=1,2$ are given by (3.11).

For this case we obtain the following eigenvalue equations

$$
\begin{aligned}
& \left(E_{2 \omega+1}-Q-Q^{-1}\right) A_{1}\left(k_{1}, k_{2}\right)=\epsilon A_{1}\left(k_{1}-1, k_{2}\right)+\epsilon A_{1}\left(k_{1}+1, k_{2}\right) \\
& \left(E_{2 \omega+1}-Q-Q^{-1}\right) A_{2}\left(k_{1}, k_{2}\right)=\epsilon A_{2}\left(k_{1}, k_{2}-1\right)+\epsilon A_{2}\left(k_{1}, k_{2}+1\right)
\end{aligned}
$$


We also have two meeting conditions that arise because pseudoparticle and impurity may be neighbors (see (3.21))

$$
A_{1}(k, k)=A_{2}(k, k+2), A_{2}(k+1, k+2)=A_{1}(k, k+1)
$$

in addition to the two conditions to be satisfied at the free ends

$$
A_{1}\left(k_{1}, N\right)=0 \quad, \quad A_{2}\left(0, k_{2}\right)=0
$$

Now we try the following ansatz for the wavefunctions

$$
\begin{aligned}
& A_{1}\left(k_{1}, k_{2}\right)=\mathrm{A}_{1}\left(\theta_{1}, \theta_{2}\right) \xi_{1}^{k_{1}} \xi_{2}^{k_{2}}-\mathrm{A}_{1}\left(\theta_{1},-\theta_{2}\right) \xi_{1}^{k_{1}} \xi_{2}^{-k_{2}} \\
& A_{2}\left(k_{1}, k_{2}\right)=\mathrm{A}_{2}\left(\theta_{1}, \theta_{2}\right) \xi_{2}^{k_{1}} \xi_{1}^{k_{2}}-\mathrm{A}_{2}\left(\theta_{1},-\theta_{2}\right) \xi_{2}^{-k_{1}} \xi_{1}^{k_{2}}
\end{aligned}
$$

From (5.8) we get the energy eigenvalue

$$
E_{2 \omega+1}=Q+Q^{-1}+\epsilon\left(\xi_{2}+\xi_{2}^{-1}\right)
$$

and from $(5.9)$ and $(5.10)$ the following relations between the coefficients $A_{i}$

$$
\begin{aligned}
\mathrm{A}_{1}\left(\theta_{1}, \theta_{2}\right) \xi_{2}^{N} & =\mathrm{A}_{1}\left(\theta_{1},-\theta_{2}\right) \xi_{2}^{-N} \quad, \quad \mathrm{~A}_{2}\left(\theta_{1}, \theta_{2}\right)=\mathrm{A}_{2}\left(\theta_{1},-\theta_{2}\right) \\
\mathrm{A}_{1}\left(\theta_{1}, \theta_{2}\right) & =\mathrm{A}_{2}\left(\theta_{1}, \theta_{2}\right) \xi_{1}^{2} \quad, \quad \mathrm{~A}_{1}\left(\theta_{1},-\theta_{2}\right)=\mathrm{A}_{2}\left(\theta_{1},-\theta_{2}\right) \xi_{1}^{2}
\end{aligned}
$$

from this we get

$$
\xi_{2}^{2 N}=1
$$

as the Bethe equation of (5.12). The coefficients $A_{i}$ are determined up to a factor that is invariant under $\theta_{2} \longleftrightarrow-\theta_{2}$ as:

$$
\mathrm{A}_{1}\left(\theta_{1}, \theta_{2}\right)=\xi_{1}^{2} \xi_{2}^{-N} \quad \text { and } \quad \mathrm{A}_{2}\left(\theta_{1}, \theta_{2}\right)=\xi_{2}^{-N} .
$$

In general, for the eigenstate with $l$ impurities with parameters $\xi_{1}, \ldots, \xi_{l}$ and one pseudoparticle with parameter $\xi_{l+1}$, which lies in a sector $r$, we can write

$$
\Psi_{r}\left(\xi_{1}, \ldots, \xi_{l+1}\right)=\sum_{j=1}^{l+1}\left\{\sum_{1 \leq k_{1}<\ldots<k_{l+1} \leq N-1} A_{j}\left(k_{1}, \ldots, k_{l+1}\right)\left|\Omega_{j}\left(k_{1}, \ldots, k_{l+1}\right)\right\rangle\right\}
$$

The corresponding eigenvalue is given by (5.5), with $\xi=\xi_{l+1}$, and the ansatz for the coefficients of the wavefunctions becomes

$$
\mathrm{A}_{j}\left(\theta_{1}, \ldots, \theta_{l+1}\right)=\left(\prod_{i=1}^{l+1-j} \xi_{i}^{2}\right) \xi_{l+1}^{-N}
$$

Here we notice that the index $j$ in the wavefunctions $A_{j}\left(k_{1}, \ldots, k_{l+1}\right)$ means that the pseudoparticle is at the position $k_{l+2-j}$.

\subsection{Two-pseudoparticles}

For the sector $r=4 \omega$, beside eigenstates with impurities, we have an eigenstate with two pseudoparticles. We obtain the following eigenvalue equations

$$
\begin{array}{r}
\left(E_{4 \omega}-2 Q-2 Q^{-1}\right) A\left(k_{1}, k_{2}\right)= \\
+\epsilon A\left(k_{1}-1, k_{2}\right)+\epsilon A\left(k_{1}+1, k_{2}\right) \\
+\epsilon A\left(k_{1}, k_{2}-1\right)+\epsilon A\left(k_{1}, k_{2}+1\right)
\end{array}
$$


We have again two conditions to be satisfied at the ends of the chain

$$
A\left(0, k_{2}\right)=0 \quad \text { and } \quad A\left(k_{1}, N\right)=0
$$

In addition to this we have a meeting condition

$$
\epsilon A(k, k)+\epsilon A(k+1, k+1)+\left(Q+Q^{-1}\right) A(k, k+1)=0
$$

Now we try the ansatz

$$
\begin{aligned}
A\left(k_{1}, k_{2}\right) & =\mathrm{A}\left(\theta_{1}, \theta_{2}\right) \xi_{1}^{k_{1}} \xi_{2}^{k_{2}}-\mathrm{A}\left(\theta_{2}, \theta_{1}\right) \xi_{1}^{k_{2}} \xi_{2}^{k_{1}} \\
& -\mathrm{A}\left(-\theta_{1}, \theta_{2}\right) \xi_{1}^{-k_{1}} \xi_{2}^{k_{2}}+\mathrm{A}\left(-\theta_{2}, \theta_{1}\right) \xi_{1}^{-k_{2}} \xi_{2}^{k_{1}} \\
& -\mathrm{A}\left(\theta_{1},-\theta_{2}\right) \xi_{1}^{k_{1}} \xi_{2}^{-k_{2}}+\mathrm{A}\left(\theta_{2},-\theta_{1}\right) \xi_{1}^{k_{2}} \xi_{2}^{-k_{1}} \\
& +\mathrm{A}\left(-\theta_{1},-\theta_{2}\right) \xi_{1}^{-k_{1}} \xi_{2}^{-k_{2}}-\mathrm{A}\left(-\theta_{2},-\theta_{1}\right) \xi_{1}^{-k_{2}} \xi_{2}^{-k_{1}}
\end{aligned}
$$

Here we observe the permutations and negations of $\theta_{1}$ and $\theta_{2}$. Substituting this ansatz in (5.18) we obtain the energy eigenvalue for the sector with two pseudoparticles

$$
E_{4 \omega}=2 Q+2 Q^{-1}+\epsilon\left(\xi_{1}+\xi_{1}^{-1}+\xi_{2}+\xi_{2}^{-1}\right)
$$

The ansatz (5.21) satisfy equations (5.19) provided that

$$
\begin{array}{r}
\mathrm{A}\left(\theta_{1}, \theta_{2}\right)=\mathrm{A}\left(-\theta_{1}, \theta_{2}\right), \quad \mathrm{A}\left(\theta_{2}, \theta_{1}\right)=\mathrm{A}\left(-\theta_{2}, \theta_{1}\right) \\
\mathrm{A}\left(\theta_{1},-\theta_{2}\right)=\mathrm{A}\left(-\theta_{1},-\theta_{2}\right), \quad \mathrm{A}\left(\theta_{2},-\theta_{1}\right)=\mathrm{A}\left(-\theta_{2},-\theta_{1}\right)
\end{array}
$$

and

$$
\xi_{2}^{2 N}=\frac{\mathrm{A}\left(\theta_{1},-\theta_{2}\right)}{\mathrm{A}\left(\theta_{1}, \theta_{2}\right)}=\frac{\mathrm{A}\left(-\theta_{1},-\theta_{2}\right)}{\mathrm{A}\left(-\theta_{1}, \theta_{2}\right)}, \quad \xi_{1}^{2 N}=\frac{\mathrm{A}\left(\theta_{2},-\theta_{1}\right)}{\mathrm{A}\left(\theta_{2}, \theta_{1}\right)}=\frac{\mathrm{A}\left(-\theta_{2},-\theta_{1}\right)}{\mathrm{A}\left(-\theta_{2}, \theta_{1}\right)}
$$

Moreover, the meeting conditions are satisfied provided that

$$
\begin{array}{r}
\frac{\mathrm{A}\left(-\theta_{1},-\theta_{2}\right)}{\mathrm{A}\left(-\theta_{2},-\theta_{1}\right)}=\frac{\mathrm{A}\left(\theta_{2}, \theta_{1}\right)}{\mathrm{A}\left(\theta_{1}, \theta_{2}\right)}=\frac{1+\xi_{1} \xi_{2}+\epsilon\left(Q+Q^{-1}\right) \xi_{2}}{1+\xi_{1} \xi_{2}+\epsilon\left(Q+Q^{-1}\right) \xi_{1}} \\
\frac{\mathrm{A}\left(\theta_{1},-\theta_{2}\right)}{\mathrm{A}\left(-\theta_{2}, \theta_{1}\right)}=\frac{\mathrm{A}\left(\theta_{2},-\theta_{1}\right)}{\mathrm{A}\left(-\theta_{1}, \theta_{2}\right)}=\frac{1+\xi_{1}^{-1} \xi_{2}+\epsilon\left(Q+Q^{-1}\right) \xi_{2}}{1+\xi_{1}^{-1} \xi_{2}+\epsilon\left(Q+Q^{-1}\right) \xi_{1}^{-1}}
\end{array}
$$

Matching these conditions we get

$$
\xi_{1}^{2 N}=\frac{B\left(-\theta_{1}, \theta_{2}\right)}{B\left(\theta_{1}, \theta_{2}\right)} \quad, \quad \xi_{2}^{2 N}=\frac{B\left(-\theta_{2}, \theta_{1}\right)}{B\left(\theta_{2}, \theta_{1}\right)}
$$

and

$$
\mathrm{A}\left(\theta_{1}, \theta_{2}\right)=\xi_{1}^{-N} \xi_{2}^{-N} B\left(-\theta_{1}, \theta_{2}\right) \xi_{2}^{-1}
$$

Here we have used the usual free boundary notations

$$
B\left(\theta_{a}, \theta_{b}\right)=s\left(\theta_{a}, \theta_{b}\right) s\left(\theta_{b},-\theta_{a}\right)
$$

where

$$
s\left(\theta_{a}, \theta_{b}\right)=1+\xi_{a} \xi_{b}+\epsilon\left(Q+Q^{-1}\right) \xi_{b} .
$$


Now let us consider the eigenstates with two pseudoparticle and impurities. The energy eigenvalue is the same of the two pseudoparticles pure state. The parameters associated with impurities are embraced in the definition of the coefficients of the wavefunctions. For instance, when we have an eigenstate of two pseudoparticles with parameters $\xi_{2}$ and $\xi_{3}$ and one impurity of parameter $\xi_{1}$, the energy is given by (5.22) and the Bethe equations by (5.26), with $\xi_{1} \rightarrow \xi_{3}$ and $\theta_{1} \rightarrow \theta_{3}$. But now the wavefunctions are different

$$
\begin{gathered}
\mathrm{A}_{1}\left(\theta_{1}, \theta_{2}, \theta_{3}\right)=\left\{\xi_{1}^{4}\right\} \xi_{2}^{-N} \xi_{3}^{-N} B\left(-\theta_{2}, \theta_{3}\right) \xi_{3}^{-1} \\
\mathrm{~A}_{2}\left(\theta_{1}, \theta_{2}, \theta_{3}\right)=\left\{\xi_{1}^{2}\right\} \xi_{2}^{-N} \xi_{3}^{-N} B\left(-\theta_{2}, \theta_{3}\right) \xi_{3}^{-1} \\
\mathrm{~A}_{3}\left(\theta_{1}, \theta_{2}, \theta_{3}\right)=\xi_{2}^{-N} \xi_{3}^{-N} B\left(-\theta_{2}, \theta_{3}\right) \xi_{3}^{-1}
\end{gathered}
$$

where $B\left(-\theta_{2}, \theta_{3}\right)$ is given by $(5.28)$

\subsection{General eigenstates}

The generalization follows as in the previous cases. In a sector $r$ with

$p$ pseudoparticles, we get

$$
E_{r}=\sum_{n=1}^{p}\left[Q+Q^{-1}+\epsilon\left(\xi_{n}+\xi_{n}^{-1}\right)\right]
$$

and the Bethe equations

$$
\xi_{a}^{2 N}=\prod_{b \neq a=l+1}^{l+p} \frac{B\left(-\theta_{a}, \theta_{b}\right)}{B\left(\theta_{a}, \theta_{b}\right)}, \quad a=1,2, \ldots, p
$$

The corresponding eigenfunction can be written as

$$
\Psi_{r}\left(\xi_{1}, \ldots, \xi_{p}\right)=\sum_{k_{1}<\cdots<k_{l+p}} A\left(k_{1}, k_{2}, \ldots, k_{l+p}\right)\left|\Omega\left(k_{1}, k_{2}, \ldots, k_{p}\right)\right\rangle
$$

with

$$
A\left(k_{1}, k_{2}, \ldots, k_{p}\right)=\sum_{P} \varepsilon_{P} \mathrm{~A}\left(\theta_{1}, \theta_{2}, \ldots, \theta_{p}\right) \xi_{1}^{k_{1}} \xi_{2}^{k_{2}} \ldots \xi_{p}^{k_{p}}
$$

where the sum extends over all permutations and negations of $\theta_{1}, \ldots, \theta_{p}$ and $\varepsilon_{P}$ changes sign at each such interchange. The coefficients in the wavefunctions are given by

$$
\mathrm{A}\left(\theta_{1}, \theta_{2}, \ldots, \theta_{p}\right)=\prod_{j=1}^{p} \xi_{j}^{-N} \prod_{l+1 \leq j<i \leq l+p} B\left(-\theta_{j}, \theta_{i}\right) \xi_{j}^{-1}
$$

where $B\left(-\theta_{j}, \theta_{i}\right)$ are defined in (5.28).

For a sector $r$ with $l$ impurities with parameters $\xi_{1}, \ldots, \xi_{l}$ and $p$ pseudoparticles with parameters $\xi_{l+1}, \ldots, \xi_{l+p}$ the energy is given by (5.31) and the Bethe equations by (5.32). Only the coefficients of the wave functions are modified

$$
\mathrm{A}_{j}\left(\theta_{1}, \theta_{2}, \ldots, \theta_{l+p}\right)=A_{j}\left(\xi_{1} \xi_{2} \cdots \xi_{l}\right) \mathrm{A}\left(\theta_{l+1}, \theta_{2}, \ldots, \theta_{l+p}\right)
$$

The functions $A_{j}\left(\xi_{1} \xi_{2} \cdots \xi_{l}\right)=\xi_{1}^{a_{1}} \xi_{2}^{a_{2}} \cdots \xi_{l}^{a_{l}}$ where the index $j$ characterizes the possible configurations of $l$ impurities relative to the $p$ pseudoparticles. Here $a_{i}$ are numbers which depend on the position of corresponding impurity relative to the pseudoparticles.

Here we observe again the valid of these results for all Temperley-Lieb spin chain Hamiltonians defined as projector of spin zero on the representations of the quantum groups $\mathcal{U}_{q}\left(X_{n}\right)$, characterized by the values of $Q+Q^{-1}=\operatorname{Tr}_{V_{\Lambda}}\left(q^{-2 \rho}\right)$. 


\section{Conclusion}

We have applied in a systematic way the coordinate Bethe ansatz to find the spectra of a series of "spin "Hamiltonians arising as representations of the Temperley-Lieb algebra. We consider several boundary conditions in order to include all previously known cases.

Due to $\mathcal{U}_{k}$ be a projector of spin zero, there is a linear combination of eigenstates of $\mathbf{S}_{T}^{Z}=\sum_{k} \mathbf{S}_{k}^{Z}$ where $\mathbf{S}_{k}^{Z}=\operatorname{diag}(\omega, \ldots,-\omega), \omega=\max (J)$, which beside simplify the calculus permits us a unified treatment for all models. We find that for a given set of boundary conditions, all models have equivalent spectra, i.e. they differ at most in their degeneracies. Moreover, for the closed cases, the spectra of the lower-dimensional representations are entirely contained in the higher-dimensional ones (see Eq.(3.23)).

Here we notice that this spectrum equivalence is, of course, a consequence of the TL algebra. Nevertheless there is in the literature a large class of Hamiltonians which are not derived from representations of the TL algebra which share the same property. The authors of reference [36] developed a technique for construction of spin chain Hamiltonians which affine quantum group symmetry whose spectra coincides with the spectra of spin chain Hamiltonians which have non-affine quantum group symmetry.

The energy eigenvalues are given by

$$
\begin{aligned}
E & =\sum_{n=1}^{p}\left(Q+Q^{-1}+2 \epsilon \cos \theta_{n}\right) \\
Q+Q^{-1} & =\operatorname{Tr}_{V_{\Lambda}}\left(q^{-2 \rho}\right)
\end{aligned}
$$

where $\rho$ is half the sum of the positive roots of $\mathcal{U}_{q}\left(X_{n}\right), \quad X_{n}=A_{1}, B_{n}, C_{n}$ and $D_{n}$. $\theta_{n}$ are solutions of the Bethe ansatz equations (3.73), (4.50) and (5.32).

The Hamiltonians for the cases $X_{n}=B_{n}, C_{n}$ and $D_{n}$ appear to be new although due to the TemperleyLieb equivalence [2], they are expected to possess the same thermodynamic properties as the $A_{1}(s=1 / 2)$ case, i.e., the spin-1/2 XXZ chain with appropriate coupling.

There are several issues left for future work. In particular, one would like clarify from an algebraic point of view, the equality of the spectra, for instance, of the biquadratic model $\left(A_{1}, s=1\right)$ and $\mathrm{XXZ}$ model $\left(A_{1}, s=1 / 2\right)$ for free boundary conditions and the inclusion of the XXZ spectrum in the one of the biquadratic Hamiltonian for periodic boundary conditions. Furthermore, the completeness and complete characterization as highest weight states of the Bethe ansatz eigenstates here presented are not considered.

Using our solutions, one can derive partition functions in the finite-size scaling limit and find the operator content of the systems constructed from these quantum chains.

Finally, we remark here that although the Hamiltonian (4.1) is a global operator, it manifests the property of essential locality [34]. From the physical point of view, this type of models exhibit behavior similar to closed chains with twisted boundary conditions, however now the boundary conditions become sector dependent.

Acknowledgments: We would like to thank A. Lima-Santos for helpful comments. This work was supported in part by CAPES-PICD, Conselho Nacional de Desenvolvimento Científico e Tecnológico (CNPqBrazil) (RCTG), and by Fundação de Amparo à Pesquisa do Estado de São Paulo (FAPESP-Brazil) (ALM).

\section{References}


[1] H.N.V.Temperley and E. Lieb, Proc. R. Soc.A322, 251 (1971).

[2] R.J.Baxter, J. Stat. Phys. 281 (1982).

[3] M.T.Batchelor and A.Kuniba, J. Phys A: Math. Gen. 242599 (1991).

[4] V.Pasquier and H.Saluer, Nucl. Phys. B330 523 (1990).

[5] M.T.Batchelor, L. Mezincescu, R.I. Nepomechie and V.Rittenberg, J. Phys. A: Math. Gen. 23 L141 (1990).

[6] F.C.Alcaraz, M.N.Barber, M.T.Batchelor, R.J. Baxter and G.R.W.Quispel, J. Phys. A: Math. Gen. 206397 (1987).

[7] P.P.Martin and V.Rittenberg, Int. J. Nucl. Phys. A7 797 (1992).

[8] H.A.Bethe, Z. Physik 71205 (1931).

[9] E.K.Sklyanin , J. Phys. A: Math. Gen. 212375 (1988).

[10] I.Cherednik, Theor. Mat. Fiz. 6135 (1984).

[11] L. Mezincescu and R.I.Nepomechie, Mod. Phys. Lett. 6A 2497 (1991).

[12] C.Destri and H.J.de Vega , Nucl. Phys. B385 361 (1992).

[13] A.Foerster and M.Karowski , Nucl. Phys. B408 512 (1993).

[14] A.González-Ruiz , Nucl. Phys. B424 468 (1994).

[15] H.J.de Vega and A.González-Ruiz, Nucl.Phys. B417 553 (1994).

[16] R.Yue, H.Fan and N.Hou , Nucl. Phys. B462 167 (1996).

[17] M.Karowski and A.Zapletal, Nucl. Phys. B419 [FS] 567 ; J. Phys. A: Math. Gen. 277919 (1994).

[18] A.Foerster, J. Phys. A: Math. Gen. 297625 (1996).

[19] P.P.Martin Potts Models and Related Problems in Statistical Mechanics (Singapore: World Scientific, 1991).

[20] H.Grosse, S.Pallua, P.Prester and E.Raschhofer, J. Phys. A: Math. Gen. 274761 (1994).

[21] A.Lima-Santos and R.C.T.Ghiotto, J. Phys. A: Math. Gen. 31502 (1998).

[22] A.Lima-Santos, Nucl.Phys.B 522 (1998).

[23] A.Lima-Santos, I. Roditi and A.Foerster,Int.J.Mod.Phys.A13,4309 (1998).

[24] N.Y.Reshetikhin, Quantized universal enveloping algebras, the Yang-Baxter equation and invariants of links I, preprint LOMI E4 (1988).

[25] V.G.Drinfeld, ICM Proc. (Berkeley)798 (1986). 
[26] J.H.H.Perk and C.L.Schultz , Physica A122 50 (1983).

[27] T.T.Truong, J. Stat. Phys. 42349 (1986).

[28] J.H.H.Perk and F.Y.Wu , J. Phys. A: Math. Gen. 42727 (1986).

[29] A.Klümper, J. Phys A: Math. Gen. 23809 (1990).

[30] M.T.Batchelor and M.N.Barber, J. Phys A: Math. Gen. 23 L15(1990).

[31] M.N.Barber and M.T.Batchelor, Phys. Rev. B40 4621 (1989).

[32] A.Affleck, J. Phys.:Condens. Matter 2405 (1990).

[33] R.Köberle and A.Lima-Santos, J. Phys A: Math. Gen. 29 519(1996).

[34] J.Links and A.Foerster, J. Phys A: Math. Gen. 302483 (1997).

[35] S.Pallua and P.Prester, J. Phys A: Math. Gen. 29 1187(1996).

[36] T.Hakobyan and A.Sedrakyan, Phys. Lett. B377 250 (1996). 